\title{
De l'Amérique du Nord au projet hémisphérique: les États-Unis, la clause sociale et l'art de la vertu démocratique
}

\begin{abstract}
Afef BENESSAIEH*
RESUME: Le texte suivant a pour but d'interroger les motivations américaines à promouvoir l'instauration d'une clause sociale venant garantir le respect de quelques droits fondamentaux des travailleurs au sein des accords commerciaux et financiers qu'ils contractent depuis la fin des années 1980. Nous y abordons l'ALENA et le projet d'intégration hémisphérique comme deux cas pour lesquels les États-Unis ont tenté - et tentent encore pour ce qui est de la ZLEA - d'adjoindre une clause sociale. Pour la majorité des commentateurs de la question, une telle disposition répondrait essentiellement à des motivations d'ordre protectionniste et parfois éthique, émanant surtout des groupes d'intérêt. Nous proposons plutôt une lecture de cette question axée sur les intérêts, et dans une moindre mesure, les idéaux, de politique internationale des EtatsUnis et tentons ainsi d'articuler une cohérence entre "unilatéralisme agressif " en matière économique et "élargissement démocratique "sur le plan diplomatique.
\end{abstract}

ABSTRACT: The following text examines American motives in advocating a social clause that seeks to guarantee a number of fundamental labour rights under the trade and financial agreements the U.S. has signed since the late 1980s. NAFIA and the proposed Western Hemisphere Free Trade Area are discussed as two cases where the United States has tried - and is still trying in the case of the WHFTA - to add a social clause. For most observers concerned with this issue, such a provision is essentially a response to protectionist and, in some cases, ethical motives held chiefly by special interest groups.

The author proposes a reading of this issue based on the interests and, to a lesser extent, the ideals of American international policy. He thus tries to describe how a unilaterally aggressive economic stance can be consistent with a diplomatic stance favouring the spread of democracy.

Là où le multilatéralisme piétine, le régionalisme semble compenser. Après que les parties contractantes à l'Accord sur les tarifs douaniers et le commerce (GATT) ont refusé d'acquiescer à la requête tardive des États-Unis d'insérer une clause sociale à l'Accord qui viendra clôturer le cycle de l'Uruguay, ceux-ci ont continué d'alimenter le débat chaque fois que l'occasion formelle s'est présentée, mais toujours pour voir celle-ci reportée à des discussions ultérieures. Comme il s'agit d'un enjeu des plus sensibles au niveau multilatéral,

\footnotetext{
* Groupe de recherche sur l'intégration continentale, Département de science politique, Universitê du Québec à Montréal. L'auteure tient à remercier particulièrement Christian Deblock et Dorval Brunelle pour leur prélecture attentive et critique des versions préliminaires de ce texte, ainsi que pour les discussions fructueuses qui ont pu en être tirées et qui ont contribué à son affinement. Les points de vue exprimés dans cet article n'impliquent toutefois que l'auteure.
}

Revue Études internationales, volume xxIx, $n^{\circ} 4$, décembre 1998 
aussi ont-ils donc opté pour une approche plus ponctuelle, là où ils sont en position d'autorité. Ainsi, deux accords "parallèles», l'un sur le travail l'autre sur l'environnement, ont-ils été adjoints à l'Alena. De même, un groupe spécial sur le travail a-t-il été constitué dans le cadre des négociations en cours en vue de mettre sur pied une zone de libre-échange dans les Amériques.

Le projet d'établir une clause sociale multilatérale, qui aurait pour objet de sanctionner entre autres l'irrespect de plusieurs normes de travail fondamentales, tels la liberté syndicale, la négociation collective, le travail carcéral et celui des enfants ainsi que les conditions de sécurité et de santé sur les lieux d'emploi, suscite en effet beaucoup d'opposition, principalement en raison de l'imprécision des motivations des États-Unis qui s'en font les promoteurs, lesquels sont alternativement, voire simultanément, soupçonnés de moralisme mal placé ou de protectionnisme insidieux. D'un point de vue économique libéral, il y a en effet peu de raisons d'être favorable à l'instauration d'une clause sociale dans les accords commerciaux, surtout s'il s'agit de protéger les pays industrialisés d'une concurrence fondée sur les faibles coûts du travail pratiqués ailleurs. Selon ce point de vue, une telle clause n'aurait d'impact que sur les secteurs d'activité reliés aux exportations et imposerait de manière aveugle des coûts supplémentaires à de nombreux pays qui n'ont pas nécessairement le niveau de développement économique suffisant pour hausser leurs standards relatifs aux conditions de travail.

Le débat sur les incidences économiques d'une clause sociale est bien couvert par une littérature récente. Du débat se dégage l'idée principale que les institutions économiques internationales ne devraient pas se mèler de questions sociales qui ont jusqu'à maintenant été prises en charge et par l'État et par des institutions spécialisées telle l'Organisation internationale du travail (orT). Cependant les dimensions politiques d'une clause sociale n'ont été que partiellement traitées. Peu d'auteurs, en effet, font le lien entre la protection sociale, la démocratisation des sociétés et l'intérêt que peuvent avoir les ÉtatsUnis à promouvoir une clause qui permettrait de mettre en place des mécanismes qui viendraient sanctionner des pratiques jugées déloyales, préciser les règles en matière de concurrence et servir de support à la démocratie. Établir ce lien permettrait de souligner le parallèle qui existe entre la consolidation de l'hégémonie américaine et l'exigence de légitimité accrue qu'elle entraîne, à quoi participerait la démocratisation à travers le monde, tout en ouvrant un questionnement sur l'intérêt qu'il y a pour les États-Unis d'avancer leur conception de la culture libérale. Bien que les motivations américaines d'ordre économique ne soient pas à écarter, ne serait-ce que parce que la menace de fermer le marché américain demeure sans conteste une rhétorique des plus persuasives, c'est davantage les incitatifs d'ordre politique, moins explorés, et certes ambivalents, qui seront creusés dans le cadre de cet article.

Une clause sociale favoriserait une meilleure représentation des travailleurs au sein de l'entreprise et de l'État en donnant voix à la main-d'œuvre pour s'associer et délibérer en vue d'améliorer ses conditions de travail. Ce processus aurait deux incidences principales: l'une, de stimuler la participa- 
tion civile en augmentant son influence, et l'autre, de mieux l'intégrer à l'organisation de la vie économique. Un droit amélioré des travailleurs, ainsi que la consolidation de classes moyennes pourvues de la capacité de participer à la vie économique, ne sont dans cette perspective que des moyens privilégiés de donner plus de légitimité aux processus de libéralisation économique en cours, ce qui, en retour viendrait conforter les États-Unis dans le rôle prédominant qu'ils entendent jouer dans la mise en place d'un ordre économique international qui doit être à la fois ouvert sur le plan économique, libéral sur le plan idéologique et centré sur eux sur le plan de la stabilité. Il y a, selon nous, une corrélation entre les incidences politiques d'une clause sociale et le dédiement renouvelé des États-Unis depuis le début des années 1990 à poursuivre une politique internationale axée sur la démocratisation. Dans ce contexte, nous croyons que la clause sociale constituerait un moyen privilégié d'opérationnaliser la stratégie d' "élargissement démocratique » par le moyen de la politique commerciale, laquelle présente l'avantage d'être éventuellement contraignante compte tenu du poids commercial et financier qui est le leur et de la latitude dont ils disposent pour sanctionner des pratiques estimées déloyales sur le plan commercial.

Le texte est divisé en quatre parties. Tout d'abord, sera précisé le contexte de politique internationale, contexte qui nous permettra de faire le lien entre le volontarisme renouvelé de la politique commerciale américaine et le projet d'«élargissement" démocratique. Ensuite, nous reviendrons sur les débats qu'a suscités au plan des institutions internationales la proposition américaine d'adjoindre une clause sociale au commerce international. Troisièmement, l'Accord nord-américain de coopération dans le domaine du travail (ANACT) sera revisité dans la perspective où il s'agit là d'une première tentative d'instaurer une clause sociale à l'échelle régionale. Enfin, le projet hémisphérique sera abordé pour mieux dégager l'accent qui est mis sur la question démocratique et la mettre en parallèle avec l'interprétation privilégiée au fil de ce texte.

\section{I-Commerce, progrès social et politique étrangère américaine}

La polémique portant sur l'opportunité d'adjoindre aux accords commerciaux une clause recouvrant quelques droits fondamentaux des travailleurs vient remettre en question l'idée centrale sur laquelle l'ordre économique international d'après-guerre a été construit, et selon laquelle le commerce allait, outre le fait inestimable de pacifier le monde, être à la source du progrès social. En effet, l'utilisation concurrentielle des normes sociales, soit leur abaissement ou leur contrôle à des niveaux inférieurs à ce que le niveau de croissance économique pourrait permettre, vient montrer que l'accélération des échanges commerciaux et financiers mondiaux et les exigences de compétitivité internationale accrue qu'elle a amplifiées, ne sont pas sans entraîner à leur suite certains effets « pervers».

Du point de vue de plusieurs économistes, une harmonisation internationale des normes de travail réduirait les gains commerciaux qui proviennent de 
la différenciation et non de la similarité des caractéristiques économiques nationales. La théorie des avantages comparatifs ${ }^{1}$ met à cet égard en lumière le fait que ce sont les disparités nationales qui alimentent le commerce et encouragent la spécialisation, principe que certains ont appliqué à la question des juridictions sociales à incidence économique, telles que les normes du travail qui contribuent au coût global de la main-d'œuvré2. L'hostilité des économistes à l'idée d'insérer une clause sociale aux échanges internationaux, s'appuie essentiellement sur un principe de neutralité voulant que le fonctionnement du marché soit laissé à sa propre régulation, tel que le souhaitaient les classiques $^{3}$. C'est la croissance économique qui serait à la source de l'amélioration des conditions de travail, ce qui disqualifie plus souvent qu'autrement l'usage des clauses sociales ${ }^{4}$. La perspective libérale affirme ainsi que

1. Dans ses Principes d'économie politique et de l'impôt (1821), David Ricardo établissait que la valeur des richesses dépendait de la «facilité ou difficulté comparative avec laquelle ces richesses sont produites $»$. Un peu plus loin et pour expliciter davantage ce qui précède, il développera sur la notion des «avantages comparés » par la parabole du vin et des draps, expliquant que les nations ont intérêt à se spécialiser dans la production qu'elles auront le plus d'avantages à échanger, parce que comparativement parlant, elle nécessite moins de coûts en terme de capital ou de nombre de travailleurs. Ricardo envisage la possibilité où la mobilité du capital fasse que le vin comme les draps puissent être produits là où ils seraient le moins coûteux, c'est-à-dire, dans son exemple, au Portugal, ce qui mettrait en question le principe des avantages comparés. Il résout le problème à l'aide d'un argument curieux, fondé sur les sentiments d'attachement à la patrie: «Nous savons cependant, par expérience, que bien des causes s'opposent à la sortie des capitaux. Telles sont: (...) la répugnance naturelle qu'éprouve tout homme à quitter sa patrie et ses amis pour aller se confier à un gouvernement étranger, et assujettir des habitudes anciennes à des mours et à des lois nouvelles. Ces sentiments (...), décident la plupart des capitalistes à se contenter d'un taux de profits moins élevé dans leur propre pays, plutôt que d'aller chercher dans des pays étrangers un emploi plus lucratif pour leurs fonds. » (...)

2. Kym Anderson, Social Policy Dimension of Economic Integration: Environmental and Labour Standards, National Bureau of Economic Research, août 1996; Alan B. KRUEGER, Observations on International Labor Standards and Trade, National Bureau of Economic Research (NBER), 5632, juin 1996; Robert Z. LAWRENCE, Trade, Multinationals, and Labor, National Bureau of Economic Research (NBER), 4836, août 1994.

3. Smith comme Ricardo s'opposaient fortement à l'intervention de l'État dans les affaires économiques qui, de leurs points de vue, devraient être régies par les lois de l'offre et de la demande. À titre d'exemple, l'un comme l'autre considérait que l'État ne devait pas employer ses ressources à soulager les indigents qui, si laissés à eux-mêmes, allaient nécessairement trouver les moyens de remédier à leur misère. Chez Smith, les dépenses d'État sont généralement taxées de dissipatoires: «Les grandes nations ne s'appauvrissent jamais par la prodigalité et la mauvaise conduite des particuliers, mais quelquefois bien par celles de leurs gouvernements. » Le principe de neutralité provient également de la fameuse métaphore du boucher de Smith concernant l'harmonie des intêrêts, cette dernière naissant non pas de la bienveillance mais de l'égoïsme de chacun, une sorte de "main invisible» faisant en sorte que la poursuite des intérêts particuliers génère le bien-être de tous.

4. Paul Krugman, «First, Do No Harm », Foreign Affairs, "Workers and Economists, the Global Economy Has Left Keynes in Its Train ", juillet-août 1996, pp. 164-170; Robert Z. LAwrENCE, "Resist the Binge», Foreign Affcirs, "Workers and Economists, the Global Economy Has Left Keynes in Its Train », juillet-août 1996, pp. 170-173; T. N. SRINIVASAN, «International Labor Standards Once Again! », dans International Labor Standards and Global Economic Integration: Proceedings of a Symposium, Washington, D.C., département du Travail, Bureau des Affaires internationales, janvier 1994. 
croissance économique et progrès social vont de pair, le principe de la rationalité économique des individus faisant en sorte que les normes sociales sont le reflet de choix sociaux dont l'exigence augmenterait dans la mouvance de la prospérité économique et de la capacité subséquente de l'État d'affecter les ressources nécessaires à l'amélioration de ces normes ${ }^{6}$.

Le courant critique a toujours remis en question cette thèse libérale de la convergence entre progrès économique et progrès social. Plusieurs soulignent l'ambiguité du lien qui existerait entre l'ouverture commerciale et la croissance économique, ainsi que la relation sans cesse plus faible entre le dynamisme de l'économie et le progrès social, particulièrement dans un contexte où l'État éprouve des difficultés croissantes à honorer ses engagements sociaux sur le plan du financement, mais aussi en raison du fait que les firmes ont la possibilité de se soustraire de leurs obligations sociales en déplaçant leurs activités dans un autre pays ${ }^{7}$. De nombreux auteurs défendent ainsi l'idée qu'une clause sociale permettrait d'empêcher que le commerce ne soit un facteur de nivellement par le bas ("social dumping»). Une telle clause aurait le double effet d'éliminer certaines pratiques abusives dans les pays en développement, et aussi de réduire les pressions à la baisse sur les conditions de travail dans les pays industrialisés sous le prétexte que ceux-ci doivent être concurrentiels ${ }^{8}$. De ce point de vue éthique, une disposition sociale insérée dans les accords commerciaux contribuerait à soulager la vie des travailleurs et réaffirmerait l'idée que c'est l'économie qui doit être au service du progrès social et non le contraire ${ }^{9}$.

5. K. ANDERSON, op. cit.; R. LAWRENCE, op. cit.

6. Banque mondiale, "Labor Standards and International Trade", Workers in an Integrating World, Oxford University Press, World Development Report 1995, p. 77.

7. Dani RoDrik, "Sense and Nonsense in the Globalization Debate», Foreign Policy, été 1997, p. 25; Frédérique SACHWAld, "Mondialisation et systèmes nationaux", dans Les défis de la mondialisation, Innovation et concurrence, Paris, Masson, 1994, pp. 14-15. Voir également les thèses de Ronald Rogowsk1, Commerce and Coalitions: How. Trade Affects Domestic Political Alignments, Princeton (N.J.), Princeton University Press, 1989, concernant les pressions du système commercial multilatéral sur les politiques nationales.

8. Sur le « dumping social » consulter Jeff FAUX, «Labor in the New Global Economy ", Dissent, été 1990, pp. 376-382; Ethan KAPSTEIn, "Workers and the World Economy", Foreign Affairs, mai-juin 1996, pp. 16-37; Jim STANFORd, Going South: Cheap Labor as an Unfair Subsidy, Ottawa, Centre canadien pour les politiques de rechange, décembre 1991; Jadish BHAGwati et Vivek H. DeHEjIA, "Freer Trade and Wages of the Unskilled - Is Marx Striking Again? ", dans J. Bhagwatl et Marvin H. Kosters (dir.), Trade and Wages: Leveling Wages Down? Washington, AEI Press, 1994, pp. 36-75; Jadish BHagwatl, « Policy Prospectives and Future Directions: a View From Academia ", dans International Labor Standards and Global Economic Integration: Proceedings of a Symposium, Washington, D. C., département du Travail, Bureau des affaires internationales, janvier 1994.

9. Jean-Marie SERVAIS, «La clause sociale dans les traités de commerce: prétention irréaliste ou instrument du progrès social?», Revue internationale du travail, 4, 1989, pp. 463-473; Gilsbert Van LIEMT, «Normes minimales du travail et commerce international: une clause sociale serait-elle opérante?", Revue internationale du travail, 4, 1989, pp. 475-492. Voir aussi les publications de l'International Labor Right and Education Fund sur la question, tels Terry CollingsworTH, J. William Goold et Pharis J. HARVEY, "Labor and Free Trade, Time for a Global New Deal », Foreign Affairs, janvier-février 1994, pp. 8-13. 
La plupart des auteurs ayant examiné l'opportunité d'adjoindre une clause sociale au commerce international ont emprunté une perspective essentiellement économique, avec le résultat que le débat a rarement dépassé le stade de la dénonciation du protectionnisme chez les uns et du plaidoyer éthique chez les autres. Quelques auteurs ont toutefois développé une troisième perspective en faisant valoir l'idée que la question des pratiques reliées aux conditions de travail était moins une question économique qu'un débat politique autour du rôle que devait jouer l'État en matière de législations du travail. Brand et Hoffmann (1994) soutiennent à cet égard que l'affaiblissement des normes sociales est surtout relié aux politiques pratiquées par les États concernés, lesquels relâchent ou durcissent davantage leurs législations pour des raisons plus ou moins contestables de compétitivité sur les marchés extérieurs ${ }^{10}$. Plusieurs soutiennent ainsi le projet d'une clause sociale pour son potentiel politique, qui est d'encourager un certain activisme social contribuant à la démocratisation des systèmes politiques des pays en développement $^{11}$, ce qui ouvrirait la possibilité d'un progrès social qui ne soit plus bridé par les intérêts politiques et économiques nationaux ${ }^{12}$.

L'engagement des États-Unis à vouloir adjoindre un volet social aux accords commerciaux remet en question la perspective optimiste que se font les économistes des incidences sociales du commerce. Cet engagement montre en effet que même en contribuant à l'amélioration des niveaux de vie et en étant un important facteur de croissance économique, le commerce peut participer au recul et à l'assouplissement des politiques de main-d'œuvre pour des motifs de compétitivité nationale, ce qui encourage davantage le maintien dans certains pays en développement d'une certaine forme d'autoritarisme

10. Diana BRAnd et Ralf HOFFMAnN, "Le débat sur l'introduction d'une clause sociale dans le système commercial international : quels enjeux? », Problèmes économiques, novembre 1994, pp. 5-13.

11. Robert Howse et Michael J. TrżiLCOCK, «The Fair Trade Free Trade Debate: Trade, Labor and the Environment», International Review of Law and Economics, 16, 1996, p. 67, écriront à cet égard: "There may be possible longer-term impacts of the reduction in oppressive labor practices that would have positive impacts on global welfare, such as accelerated political liberalization as workers become less intimidated, better organized and generally more capable of asserting their rights. »

12. Consulter aussi les travaux de Steve Charnovirz, «Labor Rights Overseas », Monthly Labor Review, aout 1995, pp. 77-78; "Environmental and Labor Standards in Trade ", The World Economy, 15 (3), 1992, pp. 335-356; «L'influence des normes de travail sur le système du commerce mondial, Aperçu historique $»$, Revue internationale du travail, septembre-octobre 1987, pp. 635-657. 
plutôt que la démocratisation des institutions politiques ${ }^{13}$. Ces considérations sont ultimement combattues par les syndicats et les organisations sociales internationales, lesquels ont œuvré depuis des lustres pour relever les conditions de travail dans le monde et faire pression contre les régimes autoritaires. Toutefois, ce sont moins des considérations éthiques et de nouvelles sympathies pro-syndicales que des raisons de stratégie commerciale d'une part, de promotion du modèle politique démocratique d'autre part, qui poussent les États-Unis à vouloir intervenir sur les pratiques sociales de leurs partenaires commerciaux. Le soutien généralisé des syndicats et autres groupes de pression sociaux à l'insertion de mesures sociales contraignantes dans les accords commerciaux, telles les normes du travail, apparaît en effet plus comme relevant de l'opportunisme politique que comme une variable explicative. Cet opportunisme permet, en effet, de justifier sur la base des contraintes de politique domestique le renforcement des sanctions commerciales, et de s'assurer ainsi l'appui de ces groupes dans la conduite de la politique commerciale, grâce à quoi l'exécutif se trouve en meilleure position pour signer des ententes commerciales qui donnent une position d'autorité aux États-Unis.

Instaurer une clause sociale multilatérale permettra aussi aux États-Unis de faire le lien entre commerce et démocratie, et de donner ainsi une cohérence et une légitimité plus grandes à une politique internationale qui doit concilier intérêts de puissance, intérêts commerciaux et valeur libérales. D'une part, sa reconnaissance et sa sanction dans les accords commerciaux autoriseraient l'intervention politique en vue de mieux niveler les conditions de la concurrence internationale (level playing field) et d'empêcher ainsi que les faibles coûts du travail ne soient utilisés de manière déloyale aux fins de compétitivité. D'autre part, et au niveau du discours, le projet d'une clause sociale s'inscrit dans le cadre d'un parti pris plus large pour la démocratisation et la défense de la liberté dans le monde, arguments de la bonne conscience pour lesquels les États-Unis ont justifié nombre de leurs interventions à travers le monde.

La rhétorique du commerce loyal, ainsi que les modifications apportées à la loi sur le commerce en 1987 pour la rendre plus contraignante s'inscrivent dans le cadre d'une politique d'« unilatéralisme agressifi ${ }^{14} »$ visant à ouvrir

13. Sur l'analyse des liens entre libéralisation économique et démocratie, toute une littérature tend à montrer que la complémentarité n'est pas nécessairement la règle. Consulter Albert BERRY, "The Income Distribution Threat in Latin America ", Latin American Research Review, 32 (2), 1997, pp. 3-40; J. F. Helliwell, «Empirical Linkages Between Democracy and Economic Growth», British Journal of Political Science, 24, 1994, pp. 225-248; S. M. LIPSET, "The Social Requisites of Democracy Revisited", American Sociological Review, 59, 1994, pp. 1-22; D. RueschmeYer et al., "Capitalist Development and Democracy: The Controversy", dans Capitalist Development and Democracy, Chicago, University of Chicago Press, 1992, pp. 12-39; L. WHITEHEAD et al., «Economic Liberalization and Democratization: Explorations of the Linkages *, World Development, $\mathrm{n}^{\circ}$ spécial, août 1993, pp. 67-92.

14. L'expression vient de Jadish BHAGWATl, "Agressive Unilateralism: An Overview», dans J. BhaGWatl et Hugh T. Patrick (dir.), Agressive Unilateralism, America's 301 Trade Policy and the World Trading System, Ann Arbor, Michigan, The University of Michigan Press, 1990, pp. I48. 
davantage les marchés extérieurs aux produits américains tout en accordant l'accès au marché américain sur la base d'une conditionnalité fondée sur les principes de bonne conduite réciproque ${ }^{15}$. La loyauté commerciale n'a rien de moral, quoi qu'en disent le Département d'État américain et les agences fédérales reliées au commerce. L'expression réfère davantage à la réciprocité et à l'acceptation de quelques principes généraux qui visent à empêcher que le jeu de la concurrence ne soit vicié par des prix artificiellement abaissés par des politiques internes répressives. C'est moins le caractère immoral de ces pratiques qui pose problème, que le fait que les pays qui recourent à ces pratiques connaissent des succès à l'exportation incontestables, lesquels ne sont pas nécessairement compensés par une croissance comparable des importations en provenance des pays industrialisés. La compétitivité fondée sur la faiblesse des pratiques sociales justifierait ainsi la nécessité de formuler des règles concurrentielles qui soient par tous observées ( «level playing field») et ainsi de s'assurer de ce que les marchés extérieurs soient aussi ouverts à la pénétration américaine que ne l'est le marché américain aux produits étrangers ${ }^{16}$. L'em-

15. L'élargissement de la section 301 de l'Omnibus Trade and Competitiveness Act (orCA) a essentiellement renforcé la capacité des États-Unis de sanctionner les pratiques commerciales «déloyales». Il incombe principalement au Bureau du Représentant au commerce (Ustr) de procéder à la réception des plaintes, aux enquêtes et aux recommandations - la décision de sanction ne pouvant émaner que du Président des États-Unis. Jusqu'à maintenant, la Super 301 n'a été utilisée que de manière incitative et aucun partenaire commercial ne s'est vu imposer des sanctions sur la base de la Loi sur le commerce. L'entérinement de la Super 301 a cependant permis de revoir certains accords commerciaux préférentiels auxquels des clauses « 301 », couvrant les droits fondamentaux de la main-d'ceuvre, ont été ajoutées, tels le renouvellement du Système des préférences généralisées (SPG) en 1988 et la Loi sur les investissements privés d'outre-mer (OPIC) en 1985. Jusqu'à présent, une dizaine de pays se sont vus retirer les avantages liés à l'attribution du SPG en raison de violations reconnues de droits de la main-d'œuvre. La plupart ont été réinstallés après l'indication de progrès réalisés à ce sujet, le dernier cas, réglé en janvier 1998 ayant porté sur le Guatemala. L'opic, administrée par l'Agence pour la garantie des investissements multilatéraux, supprime en principe la protection pour les investissements réalisés dans des pays faisant mauvaise figure en termes de respect des droits fondamentaux des travailleurs. Il n'existe pas encore de cas de retrait de la garantie des investissements pour des raisons de violation des droits de la main-d'œuvre qui puisse être rapporté. Sur les différentes législations et ententes commerciales américaines pourvues d'un volet social, voir Jorge F. PEREZ-Lopez, «Conditioning Trade on Foreign Labor Laws: the U.S. Approach», Comparative Labor Law Journal, 9 (2), hiver 1988, pp. 253-292

16. Au sujet du "Level playing field» et du dédiement conséquent des États-Unis à l'ouverture des marchés extérieurs au tournant de la fin des années 1980 - avec l'administration Bush (et son «plan de renouveau économique » axé sur le commerce international), suivie de près, en ce qui concerne l'axe international, par l'administration Clinton, survenant à la fin des négociations de l'Uruguay- on pourra lire une littérature nombreuse. A titre introductif, voir l'ouvrage très complet de BHagwati et Patrick, op.cit. Sur l'importance réaffirmée du commerce pour les États-Unis et la nécessité d'ouvrir les marchés extérieurs, lire aussi diverses livraisons du us Department of State Dispatch, telles que Mickey KanTor, " Ls Trade Policy and the Post-Cold War World ", Dispatch, 4 (1), 15 mars 1993, p. 14; President Clinton, «American Leadership and Global Change», Dispatch, 4 (9), $1^{\text {er }}$ mars 1993, pp. 116-118, discours dans lequel le Président annonce les cinq points majeurs de la politique économique américaine et souligne l'importance de la libéralisation accrue du commerce international; voir aussi Joan E. SPERO, « LS Global Economic Leadership in the Post Cold War Era», Dispatch, 6 (16), 17 avril 1995, p. 307. 
pressement américain à vouloir établir une clause sociale multilatérale participe donc de cette volonté de préciser les règles du jeu commercial international ${ }^{17}$, avec le résultat que si les États-Unis doivent continuer de se faire les porte-voix du libre-échange dans le monde, ce ne doit pas être à leurs dépens et encore moins au détriment de leur industrie. Dans cette perspective, l'argument du fair trade, identifié à tort par certains commentateurs comme témoignant d'un regain protectionniste dans la conduite de la politique commerciale américaine, ne constitue qu'un argument supplémentaire pour ouvrir davantage les marchés extérieurs ${ }^{18}$. Elle ne signifie pas, loin de là, la fermeture accrue du marché américain mais plutôt une exigence d'ouverture et de réciprocité sous la contrainte de sanctions.

Dans cette perspective, les motivations de politique commerciale à vouloir sanctionner davantage le commerce international ont plus à voir avec les gains que les États-Unis entendent tirer du libre-échange qu'avec le moralisme. Toutefois, il ne s'agit, selon nous, que d'un aspect de la question. Des motivations plus proprement politiques sont aussi à l'œuvre dans le projet d'instaurer une clause sociale.

En effet, le début des années 1990, marquées par la fin de la guerre froide, vont également être l'occasion pour les États-Unis de renouveler leur leadership politique au sein du systeme international ${ }^{19}$, et cela à travers les jeux d'un commerce, qui, plus ouvert, ne rend que plus interdépendantes ses composantes ${ }^{20}$. L'administration Clinton a accentué cette perspective en privi-

17. À ce sujet, le Congrès a chargé l'Administration de voir à établir une clause sociale au GATT la même année du passage de l'Omnibus trade bill en loi en 1987. Consulter les documents relatifs à la première session du $100^{e}$ congrès, dans États-Unis, $100^{e}$ congrès, $1^{e}$ session, Chambre des Représentants, section 880c, 8 mai 1987.

18. Judith GOLDSTEIN, "Ideas, Institutions and the Politics of Protectionism», dans J. GoldsTeIN et R. O. Keohane (dir.), Ideas and Foreign Policy, Beliefs, Institutions, and Political Change, Ithaca et Londres, Cornell University Press, 1993, pp. 3-30; Helen Milner, «The Political Economy of us Trade Policy: A Study of the Super 301 Provision », dans BHaGwaTl et PATRICK, op. cit., pp. 168-171; Claire TurEnNe Sjolander, "The Discourse of Multilateralism: U.S. Hegemony and the Management of Intemational Trade», dans W. S. Cox et C. T. SJOLANDER, Beyond Positivism, Boulder, Lynne Rienner, 1994, pp. 37-58.

19. Le terme d' «égémonie » aurait pu être employé, mais nous aurions dû procéder à quelques resserrements théoriques qui ne font pas consensus en ce qui conceme le thème du maintien ou du déclin de la position américaine dans le système international, ce que nous avons voulu éviter par souci de concision. Philip G. Cerny, "Political Entropy and American Decline», Millenium: Joumal of International Studies, 18 (1), 1989, p. 47, offre cependant une bonne définition du concept qui ne prête pas inutilement au débat: "Hegemony is a complex and contested concept (...). Most conceptions of hegemony have in common, however, the criterion that the leading state (or states) in the international system are not simply the strongest, but also have the capacity to set and enforce the international rules of the games. 》

20. Consulter Larry DiAmOND, Promoting Democracy in the 1990s, Washington, DC, 1995, Carnegie Commission; John Gerard RuGGIE, * The Past as Prologue? Interests, Identity and American Foreign Policy», International Security, 21 (4), printemps 1997, pp. 89-125; J. G. RUGGIE, «Third Try at World Order? America and Multilateralism after the Cold War», Political Science Quarterly, 109 (4) 1994, pp. 553-570; Howard J. WLARDA, "The Democracy Agenda in Us Foreign Policy», dans Cracks in the Consensus, Debating the Democracy Agenda in us Foreign Policy, Washington, DC, Center for Strategic and International Studies, 1997, pp. 1-18. 
légiant la nécessité d'adjoindre un volet politique plus clairement dessiné au système économique international, ce qui sera assuré par une inclinaison réaffirmée des États-Unis à se faire les promoteurs assidus de la démocratisation dans le monde dès $1993^{21}$. Le tournant en faveur de la démocratisation a été consacré par l'annonce de la stratégie d'«élargissement démocratique » du conseiller d'État à la sécurité nationale, Anthony Lake, lequel a déclaré en 1993 que l'kélargissement » de la communauté globale des démocraties à économies libérales, plus offensif, succéderait à la politique de sécurité américaine précédente du « containment ${ }^{22} »$. Cette stratégie remet à l'ordre du jour le rôle central des États-Unis au sein du système international, ainsi que la nécessité d'en actualiser les fondements afin de raviver la légitimité sociale sur lequel il devrait reposer ${ }^{23}$. Dans la perspective ainsi défendue, la démocratie serait le seul système politique apte à réguler les économies de marché, à favoriser leur développement et aussi à bâtir un support politique favorable au libéralisme économique au sein de la société civile ${ }^{24}$. Le projet d'instaurer une

21. L'engagement américain à défendre la démocratisation dans le monde est loin d'être nouveau. Ce qui l'est davantage, c'est la question de l'opportunité historique s'offrant aux États-Unis de défendre les principes démocratiques dans le monde dès la chute du mur de Berlin et la désagrégation du bloc soviétique, soit à partir de 1989. Voir la déclaration du Secrétaire d'État, James Baker, « us Must Act as Catalyst for Spread of Democracy », Text, 92-21, 22 avril 1992, p. 2: "Today, in the post-cold-war era, we face a third summons to leadership. This time is not about winning a war, hot or cold, but about winning a democratic peace. And this time is not about winning a peace for half a world, the free half, but for the whole world. » Voir aussi le discours de Bill Clinton, "A New Covenant for American Security», Text, 92-50, 13 novembre 1992, p. 1: "We face today the same challenge that we faced in 1946-to build a world of security, freedom, democracy, free markets and growth at a time of great change. "Voir aussi Deputy Secretary Talbott, "American Leadership in the Post-Cold War World", Dispatch, $6(18), 1^{\text {er }}$ mai 1995, p. 373 : «We face historic opportunities, not just to combat threats and enemies, but also to build a world that reflects our ideals and promotes our interests (...)». Voir aussi note suivante.

22. "From Containment to Enlargement», Remarques faites par Anthony Lake, Assistant au Président pour les Affaires de sécurité nationale, School of Advanced International Studies, Université Johns Hopkins, 21 septembre 1993. La présentation est reproduite dans Text, "National Security Advisor Lake Says us Interests Compel Engagement Abroad», 93-34, 24 septembre 1993, pp. 4-5; U.S. Department of State Dispatch, "From Containment to Enlargement », 27 septembre 1993, 4 (39), pp. 658-664; U.S. Department of State Dispatch, "A Strategy of Enlargement and the Developing World », 4 (43), 25 octobre 1993, pp. 748751. Voir aussi Douglas BRINkLEY, "Democratic Enlargement: The Clinton Doctrine", Foreign Affairs, printemps 1997, pp. 111-127; President Clinton, «A New Covenant for American Security», Text, 92-50, 13 novembre 1992, pp. 6-10.

23. La question des fondements sociaux de l'autorité politique intemationale a notamment été explorée par J. G. RuGGiE, "International Regime, Transaction and Change: Embedded Liberalism in the Post-War Economic Order », International Organization, 36 (2), 1982, p. 382.

24. Le discours de Lake accompagnant l'annonce de la stratégie d'élargissement démocratique renforce cette idée: "We see individuals as equally created, with a God-given right to life, liberty and the pursuit of happiness. So we trust in the equal wisdom of free individuals to protect those rights: through democracy, as the process for best meeting shared needs in the face of competing desires, and through markets, as the process for best meeting private needs in a way that expands opportunity. * Et, plus loin, plus intéressant encore: (...) "markets alone can expand wealth but not that sense of justice without which civilized societies perish.» ("From Containment to Enlargement», U.s. Department of State Dispatch, 4 (39), 27 septembre 1993, pp. 658-659) 
clause sociale multilatérale est lié à cette stratégie de démocratisation dans la mesure où la capacité accrue des travailleurs d'exiger de meilleures conditions de vie ainsi qu'un droit de regard sur les institutions politiques contribuerait à stimuler une meilleure représentation de leurs intérêts au sein des appareils politiques $^{25}$ et donc à favoriser leur ouverture démocratique ${ }^{26}$. La clause sociale présente aussi la particularité de favoriser le relèvement des normes sociales par l'action des acteurs sociaux concernés, c'est-à-dire les travailleurs, et ne laissent pas aux seuls gouvernements la responsabilité de voir à ce progrès social. On retrouve ainsi l'idée pragmatique selon laquelle la démocratie est, plus fondamentalement et avant d'être une institution politique, un ensemble de pratiques sociales reliées à la participation de la société civile ${ }^{27}$ aux affaires publiques

Cela étant, les motifs de politique internationale ne tombent pas du ciel. Le discours des États-Unis sur la démocratisation dans le monde n'exclut pas pour autant les motivations plus pratiques reliées à des contraintes de politique domestique dans la mesure où l'ouverture commerciale ne va pas sans susciter des appréhensions au sein de la société américaine, lesquelles sont traduites par des pressions favorables à une protection accrue du marché américain. L'administration présidentielle a toutefois de la difficulté à répondre sur ce terrain glissant; elle ne peut donner raison aux avocats d'un protectionnisme accru si elle veut continuer de présenter le projet d'une clause sociale sous son aspect plus attrayant d'instrument de démocratisation au service des sociétés civiles. Il est clair que la voix de la vertu présentant le libre-échange comme un véhicule du progrès social dans le monde est plus persuasive que la rudesse d'un tout autre discours voulant que les États-Unis

25. Lors de le Conférence annuelle de l'ort (Conférence internationale du travail, "Compte rendu provisoire», Genève, $81^{\mathfrak{e}}$ session, $7^{\mathrm{e}}$ séance, 9 juin 1994 , pp. 9/4), le Secrétaire au travail, Robert Reich, avait présenté la clause sociale de cette perspective: "Moins un pays est démocratique, plus on peut être amené à soupçonner que les normes de travail ont été supprimées pour servir des intérêts commerciaux étroits ou une impulsion mercantiliste dévoyée de la part des élites. Lorsqu'il y a des institutions démocratiques suffisamment solides, on peut présumer que les conditions de travail reflètent ce que le pays peut se permettre étant donné son niveau de développement. "

26. Sur les liens entre le développement des droits fondamentaux des travailleurs et la démocratisation ou consolidation démocratique, voir la synthèse de Samuel J. Valenzuela, "Labor Movements in Transition to Democracy», Comparative Politics, 21, juillet 1989, pp. 445-472, qui propose une discussion des travaux de G. O'Donnell et P. Schmitter sur les facteurs participant à démocratiser les appareils politiques, dont l'existence de syndicats.

27. Larry Diamond, Juan J. KInz et Seymour Martin LiPSET, "Comparing Experiences with Democracy ", dans Politics in Developing Countries, Comparing Experiences with Democracy, Boulder et Londres, Lynne Rienner Publishers, 1990, pp. 6-7, proposent des éléments de définition de la démocratie, laquelle serait un système de gouvernement reposant sur trois conditions: (i) l'existence d'une compétition significative entre les individus et les groupes organisés (dont les partis politiques) pour l'accès au pouvoir gouvememental sur un intervalle régulier et excluant l'usage de la force; (ii) une participation politique inclusive dans la sélection des chefs politiques et des politiques pratiquées ne permettant pas l'exclusion de groupes sociaux majeurs; et (iii) un niveau de liberté civile et politique (liberté d'expression, de presse, d'association) suffisant pour assurer l'intégrité de la compétition politique ainsi que de la participation. 
puissent sanctionner la concurrence étrangère tout en s'accordant un accès élargi aux marchés internationaux.

Bien qu'elle ait été chargée formellement par le Congrès de s'assurer de l'établissement d'une clause sociale au GATT $^{28}$, l'administration présidentielle ne peut plus actuellement compter sur cet appui dans ses démarches dans ce sens. Aussi se trouve-t-elle dans la curieuse position de défendre sur le plan international un projet qui fait l'objet de discordances majeures au niveau de la politique domestique, ce qui n'est pas sans lui accorder un certain discrédit. Le lien plus visible entre les lobbies syndicalistes et les Représentants d'allégeance démocrate, ainsi que la présence dominante du parti au sein des paliers législatif et exécutif pendant les trois premières années de l'administration Clinton, expliquent sans doute l'impulsion majeure qui a été donnée, en 1994, à la mise en cuvre d'une politique commerciale qui, pour être agressive, n'en faisait pas moins la promotion du respect des droits des travailleurs. Depuis 1996 toutefois, l'ambiance au Congrès s'est raidie et l'administration est dans la situation délicate de ne pouvoir abandonner ce projet, ce qui lui enlèverait l'appui de son propre parti, tout en n'étant pas autorisée par la double majorité républicaine au sein du Sénat comme de la Chambre des Représentants à aller véritablement de l'avant sur ce dossier. Le Congrès, en fait, tergiverse autant sur la question que ne le fait l'administration ellemême ${ }^{29}$, avec l'effet principal qu'il n'est plus très clair de savoir si l'administration continue de pousser de l'avant son projet, ou si elle ne fait que naviguer entre les différentes positions qui s'expriment à ce sujet au Congrès.

Pour finir, et même s'ils paralysent momentanément le projet, les vifs débats qui se déroulent au sein du Congrès sur l'opportunité d'accorder ou non à l'administration la latitude nécessaire pour qu'elle inscrive les clauses sociales à l'agenda commercial pourraient paradoxalement contribuer à sa

28. La dernière recommandation issue du Congrès date de 1994. Voir « Safeguards ", Congressional Quarterly Almanac 1994, p. 132 : [We, member of Congress] « Require the president to seek the establishment within wTo of a working party on the linkage between trade and internationally recognized worker's rights, and to report to Congress within a year of enactment on progress toward establishing the working party. * La proposition n'ayant pas été retenue au GATT, le Président n'a pu faire de rapport au Congrès sur l'établissement du groupe de travail demandé.

29. Dans une tentative de mieux préparer le passage du Fast Track en automne 1997, l'administration a été jusqu'à proposer à différentes reprises de retirer ses recommandations précédentes d'insérer des clauses sociales et environnementales aux négociations commerciales futures et de les remplacer par une « déclaration parallèle" accompagnant le texte. Curieusement, la proposition a été rejetée non seulement par de nombreux Démocrates au Congrès, principalement menés par Richard. A. Gephardt, mais aussi par plusieurs Républicains qui ont fait savoir que les enjeux des normes de travail et de l'environnement devaient être adressés au sein des accords commerciaux contractés par l'administration (voir Inside NAFTA, 1 er mai 1997, p. 36 et Americas Irade, 12 juin 1997, p. 15). Pendant les mois suivants, l'administration est revenue à ses propositions initiales de faire approuver un Fast Track qui lui permette d'insérer des clauses sociales et environnementales modérées aux ententes négociées, ce qui déplaisait aux éléments démocrates les plus actifs sur la question, pour enfin reporter le vote à un moment politique plus opportun, en raison du consensus défavorable qu'elle continuait de se voir opposer au sein de son parti comme ailleurs. 
réalisation. En effet, si une clause sociale est ultimement acceptée par les partenaires commerciaux des États-Unis, c'est parce qu'ils y seront contraints sous peine de ne pouvoir signer avec eux des ententes commerciales qui seraient autrement rejetées et par l'opinion publique nationale et par le Congrès américain. Les pressions domestiques apparaissent dans cette perspective, moins comme une entrave politique qu'une ultime sanction interne servant d'argument de persuasion supplémentaire.

\section{II - Multilatéralisme commercial et normes de travail}

Les débats sur la clause sociale ne datent pas de la naissance de l'omc, puisque déjà la question de lier le commerce international au respect des normes de travail fondamentales fut posée lors des négociations commerciales d'après-guerre en vue de compléter les accords monétaires de Bretton Woods. On s'était alors entendu pour que l'Organisation internationale du commerce administre l'Accord sur le commerce, accord qui devait progressivement libéraliser les échanges dans la communauté internationale et contribuer à forger des liens économiques accrus entres les nations, la prospérité par le commerce devenant un facteur essentiel au maintien de la paix dans le monde. L'orc devait entre autres questions se charger aussi d'établir un lien entre normes sociales et commerce international ${ }^{30}$ afin de s'assurer du fait que la libéralisation des échanges ne soit pas poursuivie sans égard pour l'objectif de progrès social que devaient viser les États à l'intérieur des frontières nationales. La Charte de la Havane qui devait sanctionner la création de l'orc ne fut jamais entérinée puisque, comme on le sait, l'administration américaine, pourtant activement impliquée dans ce projet, ne parvint pas à faire approuver celle-ci par le Congrès ${ }^{31}$. L'orc ne vit donc pas le jour, et seul sera sauvé l'Accord général sur les tarifs douaniers et le commerce (GATT), accord qui deviendra ainsi le seul cadre normatif du commerce international. En dépit de ce rejet par le Congrès, et malgré les débats internes que pouvait susciter l'inclusion de clauses sociales dans des accords commerciaux, la présidence n'en continuera pas moins de promouvoir systématiquement ce projet dans toutes les rondes de négociations successives, dans les trois dernières notam-

30. États-Unis, «Proposals for Expansion of World Trade and Employment», Département d'État, publication $n^{\circ} 2411,1945$; États-Unis, «Havana Charter for an International Trade Organization », Département d'État, publication n 3206, 1948.

31. Le Congrès américain craignait alors que l'orc ne vienne, d'une part, par trop requérir la présence américaine dans les affaires intemationales, ce qui allait à l'encontre de la tradition plus isolationniste des administrations américaines précédentes, et d'autre part, ne vienne diminuer la souveraineté politique et économique du pays, en raison du vaste champ d'action reconnu à l'orc sur le plan de la régulation du commerce international. 
ment ${ }^{32}$. Ce projet suscitera toutefois toujours beaucoup d'opposition et rien de très concret ne surgira de ces négociations.

Encore récemment, en 1994, la proposition des États-Unis d'établir, à l'oмc, un groupe de travail chargé d'étudier les liens entre commerce international et normes de travail rencontrera-t-elle l'opposition massive des pays en voie de développement, parmi lesquels ceux de l'Asie du Sud-Est, l'Inde, la Chine et plusieurs représentants d'Amérique latine ${ }^{33}$. L'argument avancé était que l'enceinte de l'omc n'était pas la plus appropriée pour traiter de la question des droits sociaux, lesquels devaient être discutés dans le cadre de l'Organisation internationale du travail (OIT), seule cette dernière ayant le mandat de discuter des droits de la main-d'œuvre et de tout ce qui relève des conditions de travail. Autre argument avancé, la plupart des pays en développement craignaient que l'insertion d'une clause sociale n'autorise des sanctions commerciales injustifiées à leur égard, lesquelles viendraient surtout protéger davantage les marchés occidentaux. La hausse des coûts de production, induite par une obligation de souscrire à des droits de la main-d'œuvre plus élevés, diminuerait en effet l'avantage concurrentiel que ces pays tirent de l'abondance et du faible coût relatif de leur main-d'œuvre. Plusieurs ont aussi pu faire valoir que les motivations américaines n'étaient pas des plus désintéressées, et qu'ils ne cautionneraient pas une proposition considérée protectionniste qui viendrait par ailleurs interférer avec leur souveraineté dans le domaine des législation sociales.

32. En 1974, le Congrès recommandera l'appui actif du gouvernement américain à tout effort multilatéral allant dans le sens de l'adoption de règles internationales sur le droit de la maind'œuvre, cé qui sera réitéré au sein du GaTT en 1979 à la conclusion de la Ronde de Tokyo (1973-1979) et par la suite, à des occasions répétées à plus de quatre reprises lors du cycle de l'Uruguay (1986-1994). Les États-Unis ont demandé formellement l'inclusion d'une clause sociale au GATT dès la reprise des négociations commerciales multilatérales (1986). Dès 1987, un texte issu du Congrès américain définissant clairement les objectifs américains à promouvoir l'adoption d'une clause sociale lors de leurs négocations au GATT, avait été adopté. Cette même année, les États-Unis ont proposé l'établissement d'un groupe de travail qui étudierait les liens entre le commerce et les droits fondamentaux des travailleurs, proposition appuyée par la plupart des pays industriels, mais rejetée par la majorité des pays en développement membres du GaTT, et ainsi renvoyée aux compétences de l'orr. En octobre 1990, les États-Unis ont réitéré leur requête concernant l'établissement d'un groupe de travail, avec le même résultat. Voir GATT, Activités en 1987, Tour d'horizon annuel des travaux du GATT, Genève, juillet 1987; GATT, "Communication des États-Unis concernant le rapport entre les normes de travail internationalement reconnues et le commerce international», Activités en 1988, Tour d'horizon annuel des travaux du GATT, Genève, juin 1988, pp. 110-111; GATr, "Proposition des États-Unis concemant l'établissement d'un groupe de travail sur les normes de travail ", Activités en 1988, Tour d'horizon annuel des travaux du GATT, Genève, juillet 1990, pp. 124-125.

33. Sur les négociations du cycle de l'Uruguay, se rapporter à l'ouvrage de Jeffrey S. SCHotT, The Uruguay Round, An Assessment, Washington, 1995, Institute for International Economics. Parallelement aux négociations de l'Uruguay, les États-Unis ont mis le débat sur la clause sociale à l'ordre du jour de la $81^{\text {e }}$ Conférence internationale du travail (1994), appuyés par la France, l'Allemagne, la Belgique et l'Irlande; plusieurs autres représentants gouvernementaux tels ceux issus de l'Espagne, de l'Italie, de la Grèce et du Portugal ne se sont pas opposés au projet. 
Aussi, si l'omc fit savoir que la question d'annexer une clause sociale au GATT demeurait ouverte et qu'elle serait ajoutée à son agenda futur, ceci ne voulait pas dire grand-chose sinon que le débat allait continuer dans les couloirs de l'organisation. Le débat sera d'ailleurs vite étouffé lors de la première réunion ministérielle de l'omc à Singapour du 13 décembre 1996. La clause sociale a certes été discutée, ce qui témoigne de l'importance de l'enjeu, et fera l'objet d'une annonce spécifique, au paragraphe 4 de la Déclaration ministérielle issue de la réunion, mais ce paragraphe maintes fois cité par la suite comme témoignant des progrès du débat (ce qu'on peut fortement nuancer) n'ajoute rien de nouveau. Elle consiste en un engagement des ministres, au nom de leurs gouvernements respectifs membres du GATT, de traiter du droit de la main-d'œuvre dans l'enceinte appropriée, c'est-à-dire à l'ort et non à l'omc, et de renouveler leur désir d'observer les principes issus de l'Organisation internationale du travail ${ }^{34}$. Mais, de ce côté-là non plus, le débat n'a guère progressé.

L'année 1994 marquera le soixante-quinzième anniversaire de l'ort ; c'était loccasion de dépoussiérer son mandat et de réanimer les principes fondamentaux sur lesquels elle avait été construite. C'est ce qui explique l'enthousiasme avec lequel l'Organisation s'est lancée dans une vaste entreprise de consultation sur l'enjeu de la clause sociale, en donnant publiquement son appui aux représentants américains et à tous ceux qui voulaient discuter des clauses sociales au sein de l'Organisation. L'ort mit sur pied un Groupe de travail sur la dimension sociale du commerce international en 1995, lequel sera chargé de procéder à des consultations avec l'ensemble des organisations internationales et régionales, de compiler les différentes mesures prises par chaque institution, relativement au droit de la main-d'œuvre, et enfin de procéder à des recommandations à l'issue de la consultation ${ }^{35}$. Cependant, moins de deux ans après l'enclenchement du processus, l'ort devait officiellement prendre ses distances du projet d'inclure une clause sociale au commerce international. Le Rapport annuel du Directeur général de juin 1997 le dit explicitement: l'Organisation ne pouvait appuyer le projet, en raison principalement du fait qu'elle ne voulait pas s'associer à un mécanisme de sanction commerciale, aussi éthiques qu'en soient les finalités, parce que cela aurait été à l'encontre du mandat et du rôle que l'Organisation voulait continuer d'endosser. Par contre, l'ort annonçait qu'elle allait procéder à la distribution de «labels

34. ONC (1996), "Déclaration ministérielle de Singapour adoptée le 13 décembre 1996 », Singapour, Conférence ministérielle, WI/MIN(96)/DEC, 18 décembre 1996, paragraphe 4: "Nous estimons que la croissance économique et le développement favorisés par une augmentation des échanges commerciaux et une libéralisation plus poussée du commerce contribuent à la promotion de ces normes. Nous rejetons l'usage des normes du travail à des fins protectionnistes et convenons que l'avantage comparatif des pays, en particulier des pays en développement à bas salaires, ne doit en aucune façon être remis en question. »

35. Voir BIT (1994), La dimension sociale de la libéralisation du commerce international, Genève, Conseil d'administration, GB. 261, novembre, Groupe de travail sur la dimension sociale de la libéralisation du commerce international; BIT (1996), Aperçu des travaux d'autres organisations ou organismes internationaux relatifs aux aspects sociaux de la libéralisation du commerce international, Genève, Conseil d'administration, GB. 265. 
sociaux » nationaux qui allaient tenir compte des secteurs d'exportation comme des secteurs d'activité réservés au marché intérieur, afin d'évaluer la situation générale des travailleurs et d'ouvrir le dialogue à l'aide de ces données ${ }^{36}$. Cette initiative n'est pas banale, même si elle dénote clairement le recul de l'ort sur la scène du débat.

Malgré les réticences apparentes des organisations internationales à se prononcer sur la proposition américaine, il reste que, depuis 1994, dans toutes les institutions où les États-Unis ont une position influente, celles-ci sont tenues, tout aussi poliment que cela puisse parfois paraître, de promouvoir l'amélioration des droits du travail en contrepartie de l'assistance qu'elles apportent aux pays en voie de développement ${ }^{37}$. Cette directive officieuse est également imposée aux départements reliés aux affaires extérieures et commerciales des États-Unis, tels principalement le Bureau du Représentant au commerce (USTR) et l'Agence pour le développement international (USAID) ${ }^{38}$.

Incapables de persuader l'ensemble de la communauté internationale du bien-fondé de leur requête d'instaurer une clause sociale aux échanges

36. BIT (1997), «Commerce international et droits des travailleurs: le Directeur général du BIT préconise l'adoption de règles universelles», Communiqué de presse (BIT/97/10), 23 avril; BIT (1997), L'action normative de l'or à l'heure de la mondialisation - Rapport du Directeur général, Conférence internationale du Travail, $85^{\circ}$ session.

37. Les départements de recherche des Fonds monétaire international et de la Banque mondiale ont récemment contribué au débat sur la clause sociale en publiant chacun une note de recherche sur le sujet. Les deux études économiques ajoutent peu aux travaux de Hansson, tels que Göte Hansson, Social Clauses and International Trade, New York, St. Martin's Press, 1983, lesquels indiquaient clairement que du point de vue quantitatif, une clause sociale aurait peu d'effets positifs et contribuerait d'une part à protéger davantage les marchés occidentaux et d'autre part à créer du sous-emploi et de la pauvreté dans les secteurs d'exportation au Sud, le coût accru de la main-d'œuvre ou les restrictions normatives imposées aux firmes ayant l'effet principal de les dissuader d'embaucher davantage. Les articles de Maskus (1997) et de Golub (1997) indiquent respectivement qu'il n'existe aucune évidence sur le fait que le commerce international puisse avoir des incidences sur les pratiques sociales, et retiennent en bref le cas unique du travail des enfants comme pouvant faire l'objet d'une réglementation internationale, le travail des enfants étant surtout employé dans les industries qui nécessitent une nombreuse main-d'ceuvre et qui sont reliées de façon un peu plus visible aux secteurs d'exportation. Lire Stephen S. Golub, Intemational Labor Standards and International Trade, Fonds monétaire international, Working Paper, WP/97/ 37, avril 1997 et Keith E. Maskus, Should Core Labor Standards be Imposed Through International Trade Policy?, Washington, DC, Banque mondiale, Policy Research Working Paper, 1817. août 1997

38. L'USAID est plus particulièrement centrée sur l'Amérique latine depuis le Sommet de Miami de 1994, où l'Initiative pour les Amériques a été lancée, et travaille en collaboration avec le Bureau de la coordination du Sommet pour le Département d'État. Le dossier social dans les Amériques implique principalement l'enjeu de la «consolidation de la société civile " et l'encouragement à développer des micro-projets qui contribuent à assurer une plus grande décentralisation des ressources économiques et politiques, la lutte contre la pauvreté et la démocratisation accrue des sociétés et structures gouvernementales du continent latin. L'usaD est principalement épaulée par la Banque interaméricaine de développement pour ce qui est du support technique et des ressources d'expertise, ainsi que par certains départements de l'Organisation des États américains, telle l'Unité pour la promotion de la démocratie. 
multilatéraux, les États-Unis ont choisi de mettre à l'essai le projet sur une base régionale et hémisphérique, d'abord avec l'alénA, puis avec les dispositions qui régiraient la Zone de libre-échange des Amériques lors de sa création en 2005. Cette stratégie "compensatoire» n'est pas unique en son genre, plusieurs auteurs ayant remarqué que là où le multilatéralisme progressait trop lentement, le bilatéralisme et le régionalisme offraient certains recours, perspective qui semble particulièrement s'appliquer aux clauses sociales ${ }^{39}$. Voyons ce qu'il en est de ce côté.

\section{III - L'Accord de libre-échange nord-américain}

Nombre d'économistes ont analysé les impacts que l'AléNA aurait sur l'économie américaine; la majorité d'entre eux estiment que les incidences de l'Accord seraient à toutes fins pratiques insignifiantes, en raison surtout de la différence de taille phénoménale entre les marchés mexicain et américain ${ }^{40}$. Krugman a certainement le mieux résumé l'intérêt pour les États-Unis de contracter un tel accord: l'ALÉNA était à l'origine une question de politique étrangère et l'Accord n'avait d'autre but que de stimuler la croissance économique au Mexique pour, d'une part, raréfier les migrations illégales massives en direction des États-Unis et, d'autre part, mieux sceller les réformes économiques entreprises au Mexique depuis le début des années 198041. En théorie, la plus grande prospérité de l'économie mexicaine contribuerait par-dessus tout à la stabilité politique du pays et à l'ouverture progressive de l'appareil gouvernemental mexicain aux différents intérêts exprimés au sein de la société

39. Theresa AMATO, « Labor Rights Conditionality, U.S. Trade Legislation and the International Trade Order », New York University Law Review, 65 (79), avril 1991, p. 120; Lance CoMPA, "Labor Rights and Labor Standards in International Trade ", Law and Policy in International Business, 25 (1), 1993, p. 190.

40. Consulter Drusilla K. Brown, «The Impact of a North American Free Trade Area: Applied General Equilibrium Models », dans Nora LustiG, Barry P. Bosworth et Robert Z. LAWRENCE (dirs.), North American Free Trade, Assessing the Impact, Washington, D.C., The Brookings Institution, 1992, pp. 67-68; Jeffrey J. Schotg et Gary C. HuFbAuER, NAFTA: Issues and Recommandations, Washington, DC, Institute for International Economics, 1993; Sydney WEINTRAUB, «The North American Free Trade Agreement as Negociated : a u.s. Perspective ", dans Steven Globerman et Michael Walker (dir.), Assessing NAFTA, a Trinational Analysis, Vancouver, The Fraser Institute, 1993, pp. 1-31.

41. Voir Paul KRUGMAN, «The NAFra Debate, the Uncomfortable Truth About NAFTA: It's Foreign Policy, Stupid », dans Foreign Affairs, vol. 72, \#5, novembre-décembre 1993, pp. 13-19. 
civile $^{42}$. Évidemment, en pratique, l'expérience de l'alÉnA a été jusqu'à maintenant tout autrement, avec l'effet que l'Accord semble avoir davantage vigoureusement déséquilibré l'économie mexicaine qu'il ne l'a conduite sur une voie de croissance sereine, mais sans que les attentes d'assister à la démocratisation graduelle de l'État mexicain n'aient pour autant disparu.

On se rappellera que le président Clinton avait fait campagne en tentant de rallier modérément Républicains et Démocrates, pro et contre libreéchangistes, au sein de l'arène politique comme de l'électorat, en proposant un Accord de libre-échange amélioré par l'ajout de deux ententes "parallèles " dont l'une devait porter sur les normes de travail. L'Accord nord-américain de coopération sur le travail fut négocié et ajouté à l'ALÉNA en septembre 1993 à l'initiative de la nouvelle administration et ce, malgré le désaccord initial des négociateurs canadiens et mexicains ${ }^{43}$. À l'origine, l'administration américaine souhaitait établir une entente beaucoup plus contraignante, basée sur l'usage de sanctions, plutôt qu'une entente qui ne vise qu'à promouvoir la coopération. C'est l'opposition des négociateurs canadiens et mexicains qui amènera les États-Unis à nuancer davantage leurs propositions, mais il n'était pas envisageable de les abandonner: l'AlÉNA aurait été bloqué au Congrès si les équipes canadiennes et mexicaines n'avaient finalement accepté les ententes parallèles peu de temps avant le vote au Congrès accordant la ratification de l'AlÉNA. Celles-ci allaient permettre de gagner davantage de voix démocrates sans pour autant s'aliéner les votes républicains, lesquels appuyaient générale-

42. Delal Bafr et Sydney Weintraub, "The Pressure for Political Reform in Mexico", dans The NAFTA Debate: Grappling with Unconventional Trade Issues, Boulder, London, Lynne Rienner Publishers, 1994, pp. 159-180; Carlos A. HEREDIA, « NAFIA and Democratization in Mexico», Journal of International Affairs, été 1994, 48 (1), pp. 13-38; Stephen B. PREECE et Claudio D. MilmaN, "Pluralistic Participation in an Era of Economic Blocs: the Case of Mexico", dans Fatemi Khosrow et Dominick Salvatore (dirs.), The North American Free Trade, Oxford, Pergamon, 1994, pp. 55-64; Francisco VALDÉS-UGALDE, « The Changing Relationship Between the State and the Economy in Mexico», Challenge, mai-juin 1995, pp. 32-36; Howard J. WIARDA, "Mexico ", dans Cracks in the Consensus, Debating the Democracy Agenda in us Foreign Policy, Washington, DC, The Center for Strategic International Studies, 1997, p. 51. Pour une critique de la perspective libérale voulant que l'AlÉNA contribue à la démocratisation du Mexique, voir Ian Robinson, North American Free Trade as if Democracy Mattered, Ottawa, Canadian Centre for Policy Alternatives, septembre 1993.

43. Hermann von Bertrab, Negociating NAFTA, A Mexican Envoy's Account, Washington, DC, Center for Strategic International Studies, 1997, p. 81. Pour une présentation plus détaillée du point de vue canadien sur les négociations ayant précédé la signature des ententes, lire Dalil MASCNO, "L'Accord nord-américain de coopération cans le domaine du travail, l'ALÉNA et la clause sociale », dans Droits du travail et commerce international, textes présentés par René Laperrière, Québec, Les éditions Yvon Blais, Université du Québec à Montréal, Département des sciences juridiques, 1995, pp. 1-32, économiste principal au Secrétariat de coordination interne. 
ment l'Accord ${ }^{44}$. Finalement, l'ALÉNA fut approuvé au Congrès en novembre 1993, par 235 voix contre 199, les Démocrates ayant appuyé la ratification de l'Accord de 102 votes contre 156 et les Républicains de 132 contre 43.

La communauté des affaires a sans conteste été extrêmement favorable à la perspective de la signature de l'ALÉNA, ce dernier étant envisagé dans une perspective d'efficience accrue du fonctionnement du marché nord-américain, d'élargissement des opportunités d'affaires ainsi que de plus grande transparence dans les réglementations commerciales. Plusieurs organisations de gens d'affaires, tel le Business Roundtable, ont fait savoir lors des négociations ayant précédé la signature des ententes parallèles de l'ALÉNA leur opposition à la conclusion de toute entente comprenant un volet social trop contraignant ${ }^{45}$ et ont aussi manifesté leur désaccord à ce que la Zone de libre-échange dans les Amériques soit pourvue d'un volet social ${ }^{46}$. D'un autre côté, l'AlENA a été vivement contesté au sein de l'opinion publique par les groupes sociaux de toutes allégeances, les Organisations non gouvernementales et les syndicats ${ }^{47}$, pour la raison principale que l'intégration économique induirait des pressions à la baisse sur les conditions de travail au Nord. La ligne critique n'a toutefois pas reçu bonne presse et a été généralement perçue comme défendant les intérêts établis des travailleurs syndicalisés et ultimement protectionnistes. Aussi, le discours des groupes sociaux américains a souffert d'un discrédit que n'ont pas connu les coalitions canadiennes et mexicaines, généralement épau-

44. Les congressistes fortement opposés à l'Accord pour les impacts qu'il pourrait avoir sur l'emploi provenaient des deux partis principaux, plusieurs Républicains s'étant ralliés à l'aile ultra-conservatrice du parti, menée par le candidat aux élections présidentielles de 1992 Ross Perrot. Ce demier avait lancé une formule qui fera grand bruit réduisant l'Accord à une image de succion géante des emplois américains vers le Mexique ("the big sucking sound from the south»). Voir, pour l'attention apportée à la question de l'emploi au sein des débats du Congrès, l'étude quantitative de Leo H. KaHANe, «Senate Voting Patterns on the 1991 Extension of the Fast-track Trade Procedure: Prelude to NAFTA », Public Choice, 87, 1996, pp. 35-53.

45. Henry J. JACEK, «Public Policy and NAFTA: The Role of Organized Business Interests and the Labor Movement », Canadian-American Public Policy, 19, octobre 1994, pp. 1-30.

46. Inside NAFTA, 29 juin 1994, p. 1 ; « Letter from the National Foreign Trade Council to the us Trade Representative», Inside NAFIA, 13 juillet 1994, pp. 1-2.

47. Gordon I. Clark, « NAFTA-Clinton's Victory, Organized Labor's Loss », Political Geography, 13 (4), juillet 1994, pp. 377-384; John A. Conybeare et Mark Zinkula, «Who Voted Against NAFTA? Trade Unions Versus Free Trade», World Economy, 19 (1), 1996, pp. 1-12; Ken JENNINGS et Jeffrey W. STEAGALI, "Unions and NAFTA's Legislative Passage: Confrontation and Cover ", Labor Studies Journal, 21 (1), printemps 1996, pp. 61-79. 
lées par leurs formations politiques nationales les plus travaillistes ${ }^{48}$, la campagne d'opposition à l'Accord ayant été associée à l'aile ultra-conservatrice du parti Républicain, ce qui lui a ôté le caractère progressiste dont elle aurait pu faire montre sans cette affiliation. L'opposition à l'ALÉNA des groupes concernés ne diminuera toutefois pas avec la perspective de voir l'Accord complété par les deux ententes parallèles sur le travail et l'environnement. Celles-ci étaient perçues comme dépourvues de tout caractère véritable puisque ménageant tout un chacun sans véritablement satisfaire entièrement quiconque, et cela au sein des coalitions civiles comme au Congrès.

L'essentiel des critiques adressées à l'entente sur le travail consistait à souligner le fait que sur les onze normes de travail identifiées comme principes directeurs et que les parties contractantes s'engageaient à respecter, seules trois d'entre elles (le travail des enfants, la santé et sécurité sur les lieux de travail et les salaires) pouvaient faire l'objet de sanctions ${ }^{49}$. De ces normes sujettes à sanctions, les droits reliés à la liberté d'association et de négociation des conditions d'emplois étaient exclus, en raison du fait qu'ils interféraient avec les législations nationales mexicaines ${ }^{50}$. De plus, la procédure de règlement des différends apparaissait aussi sophistiquée qu'interminable, ce qui mettait en doute pour plusieurs l'efficience et l'utilité de l'Anact. Il peut en effet s'écouler jusqu'à 1435 jours, soit près de quatre années, entre le dépôt d'une plainte et l'établissement d'une amende. Les promoteurs de l'entente et les observateurs modérés ont toutefois souligné l'apport que pouvait constituer la Commission sur le plan de la coopération, l'échange d'informations et la plus grande visibilité des pratiques reliées aux conditions de travail, recon-

48. Voir lan RoBInson, «NAFTA, Social Unionism, and Labour Movement Power in Canada and the United States", Relations industrielles, 49 (4), 1994, pp. 673-677, pour une liste exhaustive des organisations populaires et syndicales qui se sont prononcées contre l'entente. L'action des groupes sociaux mexicains et des partis politiques critiques est rapidement décrite dans Adolpho Aguilar Zinser, "Is There an Alternative? the Political Constraints on NAFta», dans Victor Bulmer-Thomas, Nikki Craske et Monica Serrano (dir.), Mexico and the North American Free Trade Agreement, Who Will Benefit?, New York, St. Martin's Press, 1994, pp. 124-125. Pour un tableau d'ensemble des différents groupes sociaux qui se sont organisés régionalement pour s'opposer à l'entente, lire Ralph AMBRUSTER, «Cross-National Labor Organizing Strategies », Critical Sociology, 21 (2), 1995, p. 79 et suivantes.

49. L'ANACr couvre les onze principes généraux suivants: la liberté d'association, le droit à la négociation collective, le droit de grève, l'interdiction du travail forcé, l'interdiction du travail des enfants, les normes minimales d'emploi (salaire), l'égalité de rémunération entre hommes et fermmes, la prévention des maladies professionnelles et des accidents de travail, l'indemnisation en cas d'accidents de travail ou de maladies professionnelles et la protection des travailleurs migrants. Voir ANACT, Annexe 1, «Principes relatifs au travail».

50. L'ANaCt ne peut légiférer sur des normes qui ne sont pas reconnues par l'ensemble des Parties (ANaCt, Article 29 (lb)) 
naissant le fait que sa capacité de sanctionner était plus dissuasive que véritablement opérationnelle ${ }^{51}$.

L'ANACT engage essentiellement les trois États à appliquer leurs propres législations du travail et à promouvoir leur amélioration ${ }^{52}$. Les mécanismes contraignants établis dans le cadre de la Commission du travail peuvent être déclenchés à l'issue du constat de la violation d'une des trois normes identifiées plus haut ${ }^{53}$. Techniquement, la structure établie par l'Accord consiste en un Conseil des ministres du travail ${ }^{54}$, supervisant un secrétariat de coordination internationale ${ }^{55}$, qui administre à son tour trois bureaux d'administration nationale ${ }^{56}$. L'ensemble de la structure ${ }^{57}$, la Commission de coopération dans le domaine $d u$ travail, asseoit son autorité sur deux instruments. Le premier tient à l'engagement contractuel des trois parties signataires de coopérer sur les

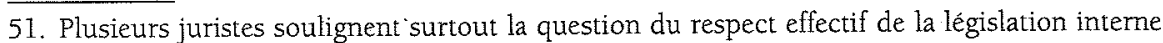
des Parties signataires qui fait partie des engagements pris dans le cadre de l'anact. Sur l'échange d'informations et la coopération établie entre les partenaires, autre avantage majeur de l'entente, voir René LAPERRIËRE, «Synthèse. Bilan, enjeux et perspectives: la commercialisation internationale des droits du travail», dans Droits du travail et commerce international, textes présentés par René Laperrière, Québec, Les éditions Yvon Blais, Université du Québec à Montréal, Département des sciences juridiques, 1995, p. 112. Sur le plan du processus de règlement des différends, les juristes qui ont été favorables à l'ANACI reconnaissent toutefois les faiblesses de l'entente. Dans l'ANACT, la sanction ne constitue qu'un ultime recours et non pas un instrument de renforcement véritablement opérationnel de l'accord. Laperriere, p. 114, estime que: "Le degré de preuve requis pour enclencher le processus de sanction, notamment celle de caractère systématique des violations, semble vouer une bonne partie des plaintes à l'échec. "

52. Voir ANACT, Articles la et 2.

53. ANACT, Articles 27 (1) et 29 (1). Le mécanisme coercitif de la Commission intervient lorsque le groupe arbitral nommé par le Conseil vérifie que la norme enfreinte l'a été de «façon systématique» (Article 29 (1), que celle-ci se rapporte au commerce (Article 29 (1a) et qu'elle est couverte par des lois du travail mutuellement reconnues (Article 29 (1b).

54. ANACT, Articles 9 à 11.

55. ANACT, Articles 12 à 14. Le secrétariat interne de coordination, assistant le Conseil ministériel, réunit, publie des informations et coordonne l'ensemble des activités de la Commission. Chaque pays dispose également d'un Bureau administratif national (BAN), qui reçoit les plaintes, les analyse et les achemine, lorsqu'acceptées, au secrétariat interne. Le mécanisme contraignant prévu par l'Accord est effectif lorsque les pays concemés par un litige ne parviennent pas, par la consultation et le dialogue, à régler ce différend. Dans ce cas, et sur vote de deux membres sur trois du Conseil, un groupe spécial fait enquête et propose un plan de correction. Enfin, si la recommandation n'est pas mise en application, une compensation monétaire est exigée. Cette compensation peut aller jusqu'à 20 millions \$us pour la première année et ne pourront pas dépasser $0,007 \%$ du total des échanges trinationaux de marchandises pour les années subséquentes. On peut résumer le processus de règlement des différends en cinq étapes distinctes: consultation entre BAN, consultation ministérielle, évaluation comparée indépendante, dernière consultation ministérielle, et enfin, recours aux procédures de règlement des différends.

56. ANACT, Articles 15 et 16.

57. L'ensemble de la Commission est établi par la Partie m de l'AnaCt, Article 8. De nombreux articles ont décrit le fonctionnement de la Commission. Parmi ceux-là, on pourra consulter les travaux de Jill SANNER RUHNKE, "The Impact of NAFTA on Labor Arbitration in Mexico», Law and Policy in International Business, 26 (3), printemps 1995, pp. 917-944. 
questions du travail et de ne pas abaisser leurs normes respectives de travail ${ }^{58}$. Le second consiste en des sanctions monétaires s'appliquant lors de violations répétées des normes couvertes par l'Accord ${ }^{59}$. Il existe peu d'études portant sur l'efficacité de la Commission à régler les différends survenant sur le respect des normes de travail couvertes par l'accord. La Commission a reçu jusqu'à présent neuf plaintes relatives aux conditions de travail au Mexique. L'une d'entre elles avait été refusée en 1995 parce que ne pouvant faire l'objet d'un examen légitime. Le cas concernait les droits syndicaux, lesquels ne peuvent être sanctionnés au sein de l'ANACT. Les deux premières plaintes auraient été rejetées par le BAN américain, tandis que les suivantes ont fait l'objet de rapports ${ }^{60}$. Les six cas reçus mais sans impliquer davantage les mécanismes de sanction de l'ANACT ont fait l'objet de recommandations, mais n'ont pas dépassé le premier palier d'intervention de l'ANACT. Ils concernaient tous des questions relatives à la liberté syndicale, lesquelles peuvent faire l'objet de recommandations mais ne sont pas susceptibles de mener à des sanctions. monétaires ou commerciales. Seul le cas relié aux tests de grossesse exigés des femmes au moment de leur embauche dans des maquiladoras mexicaines est maintenant officiellement accepté, avec l'éventualité de l'activation des procédures de sanction prévues par l'aNaCT.

La mise en ceuvre de l'áénA semble s'inscrire dans l'esprit du raffermissement de la politique extérieure américaine sur les plans du commerce et de l'incitation à la démocratisation. L'Accord favorise une stimulation des échanges commerciaux entre le Mexique et les États-Unis, mais admet certaines réserves faisant que si le marché américain est plus ouvert, les États-Unis n'entendent pas laisser le Mexique continuer de comprimer les coûts de la main-d'œuvre et concurrencer « déloyalement » leurs propres industries. L'Accord autorise grâce à l'ANACT les sanctions des pratiques reliées au traitement de la main-d'œuvre, mais en ultime recours, ce qui concorde avec le fonctionnement plus incitatif que véritablement contraignant des autres ententes issues de la Super 301. D'un autre côté, il est clair que l'élément démocratique était significatif dans le projet de contracter un Accord de libre-échange avec le Mexique qui soit flanqué d'un volet social. La situation contiguë des deux territoires mexicain et américain fait en sorte que le climat sociopolitique au Mexique ne laisse certainement pas indifférents les États-Unis, pour des raisons de sécurité et de stabilité politique de la région. L'Accord a été justifié largement pour la contribution qu'il pourrait apporter à assurer la prospérité économique du Mexique et au relèvement conséquent des niveaux de vie, ce qui participerait à réduire les migrations illégales en direction des États-Unis,

58. Sur la coopération, esprit essentiel de l'accord, voir ANACr, Partie 1, Objectifs c et e. Sur l'obligation d'améliorer les normes de travail, lire l'Article 2.

59. Lire ANACT, Partie v (Règlement des différends), et les Annexes 39 et $41 \mathrm{~B}$.

60. Pour l'exposé des premiers cas de litige, consulter l'article critique de Walt Vanderbusch, "Mexican Labor in the Era of Economic Restructuring and NAFTA: Working to Create a Favorable Investement Climate", Labor Sudies Joumal, 20 (4), 1996, pp. 58-86. Pour une vue d'ensemble de tous les cas traités dans le cadre de l'ANACT, lire "Nitlo Secretariat Summary of NAFTA Labor Case (Informal)», Americas Trade, 25 décembre 1997, pp. 9-11. 
ainsi qu'à stabiliser le pays sur le plan politique. L'ANACT a, de son côté, ajouté une exigence de transparence et de mise en circulation de l'information relative aux politiques et aux pratiques reliées à la main-d'œuvre, incitant ainsi le gouvernement mexicain à mettre en ouvre avec plus de zèle ses propres législations, ce qui constitue en outre une obligation formelle issue de l'ANACT. Cette dernière concession est majeure pour le Mexique, lequel dispose d'un code du travail élaboré que les autorités ne font pas nécessairement respecter ${ }^{61}$.

L'ANACT a offert un support institutionnel aux requêtes issues des particuliers et des regroupements sociaux sur le suivi des conditions de travail au Mexique, qui n'étaient auparavant que l'affaire des autorités concernées. La bonne nouvelle est que l'entente a accru l'influence des groupes sociaux actifs sur les droits du travail, lesquels peuvent faire pression sur le gouvernement mexicain par l'intermédiaire de la Commission, avec l'effet que si les abus au chapitre des conditions de travail n'en continuent pas moins, le gouvernement mexicain ne dispose désormais plus du même voile de discrétion et doit répondre aux demandes d'information exprimées par les représentants politiques canadiens et américains concernés. La mauvaise nouvelle est que pour tous ceux qui n'avaient vu dans l'ANaCt qu'une autre formalité juridique rendant l'ALÉNA plus acceptable auprès des coalitions civiles, il y a désenchantement. Sans être d'une efficacité renversante, la Commission n'en a pas moins été mise à contribution avec insistance, avec le résultat que le peu que recouvre l'ALÉNA en matière de droit de la main-d'œuvre apparaît déjà comme de trop comme l'ont fait savoir plusieurs des pays engagés dans le projet des Amériques.

\section{IV - La clause sociale à l'agenda des Amériques}

Depuis le début des discussions entre les trente-quatre pays participant en vue d'établir une zone de libre-échange dans les Amériques, les États-Unis ont cherché à faire avancer le dossier social pour l'inclure dans les discussions formelles $^{62}$. Idéalement, l'administration aurait voulu, dès Miami, persuader les chefs d'État de l'ensemble de l'hémisphère du bien-fondé de voir à ce qu'une clause sociale, inscrite à l'agenda de la consolidation démocratique, soit partie du projet d'intégration hémisphérique. En l'absence d'un tel résultat, seules les initiatives reliées à la promotion démocratique ont été retenues à

61. Consulter Dan LA BotZ, «Labor Rights and the Law », dans Labor Suppression in Mexico Today, Montréal, New York, Black Rose Books, International Labor Rights Education and Research Fund Book, 1992, pp. 39-60.

62. En juillet 1994 le Département d'État et le Conseil de sécurité nationale ont conjointement demandé aux trois organisations qui formeront le Comité tripartite, soit la Banque interaméricaine de développement (BID), la Commission des Nations Unies pour l'Amérique latine et les Caraibes (CÉPALC) et l'Organisation des États Américains (OÉA), d'étudier les stratégies à prendre pour développer le «dossier social » dans les Amériques et le faire adopter à Miami, faisant référence entre autres aux questions de la démocratie et de la pauvreté. Voir Inside NAFTA, 27 juillet 1994, p. 10. 
l'ordre du jour du processus enclenché, avec l'effet que les documents issus du Sommet des Amériques à Miàmi de 1994 font référence avec insistance à l'importance de voir à stimuler la participation de la société civile au sein des appareils politiques respectifs, laquelle était vivement encouragée par l'administration Clinton sous le couvert de poursuivre une «diplomatie participative ». Toutefois, le Plan d'Action et la Déclaration de Miami ne font référence que de manière très générale aux modalités de cette participation de la société civile, la Banque interaméricaine de développement et l'OÉA ayant le mandat d'y voir plus concrètement ${ }^{63}$.

Le premier Sommet des Amériques a été l'occasion d'établir clairement le fait que pour les États-Unis, la priorité du projet de créer une zLÉA était de concourir à la démocratisation accrue dans l'hémisphère, laquelle permettrait de mieux garantir le fait que les réformes économiques et politiques sont

63. Le Plan d'Action du Sommet des Amériques de 1994 accorde à la BID le rôle central en ce qui conceme la stimulation de la participation civile aux questions publiques. Depuis 1994, la BID a réorienté de façon majeure ses activités pour privilégier les dossiers sociaux, auxquels plus de la moitié de ses ressources financières sont affectées. Plusieurs départements de recherche de la BID sont impliqués sur ces dossiers par l'intermédiaire de la Division des programmes sociaux, créée en 1994, qui fournit une assistance technique à l'élaboration et la mise en ceuvre des politiques reliées à la pauvreté, l'habitation, la santé, l'éducation, entre autres questions. L'Institut interaméricain pour le développement social (iNDEs) établi en 1994 après le Sommet de Miami, foumit une assistance technique de formation pour l'élaboration et l'administration des politiques sociales. L'institut travaille avec les représentants gouvernementaux et les organismes non gouvernementaux qui ont un rôle influent dans le domaine de l'élaboration et la mise en ceuvre des politiques sociales. L'Institut pour l'intégration de l'Amérique latine et les Caraibes (INTAL) est plus orienté sur la mise à jour de recherches et données statistiques relatives aux relations économiques dans l'hémisphère. La cépalc n'a pas reçu de mandat précis dans le cadre du Plan d'Action du Sommet des Amériques issu en 1994, mais elle a notamment contribué à mettre à jour un système d'information et d'analyse relatives aux conditions de vie économique et sociale dans les Amériques. La publication annuelle de son Rapport social remplit ce rôle. Indépendamment du projet de la ZIF́A, la CÉPALC a travaillé depuis 1995 à assurer un suivi en ce qui concerne la région des Amériques au Sommet pour le développement social organisé par l'onu à Copenhague en 1995. L'OÉA, plus axée sur la diplomatie que l'intervention auprès des individus et organisations sociales, contribue cependant au dossier par l'intermédiaire de ses départements sur l'éducation, la démocratie et les droits humains. L'Unité pour le développement social et l'éducation pilote des recherches et publie des données relatives au problème de la pauvreté dans l'hémisphère et s'implique dans des projets d'éducation civile sur le terrain. L'Unité pour la promotion de la démocratie, hormis son travail d'information et de formation technique, est notamment chargée de l'envoi d'observateurs pendant les périodes électorales. La Commission interaméricaine des droits de l'homme assure les visites sur le terrain, publie des rapports sur la situation des droits humains dans l'hémisphère et fournit des recours judiciaires aux particuliers et groupes victimes de violations. Voir Robin L. ROSENBERG, Implementing the Summit of the Americas: Invigorating Civil Society Participation, Miami, Université de Miami, North-South Center, 1996; Alvaro TiRado Mejla, Integracion y Democracia en America Latina y el Caribe, Washington, DC, BID, INTAL, Documento de Divulgacion 1, 1997. 
réalisées dans un climat de stabilité sociale $e^{64}$. Une clause sociale aurait éventuellement permis de concrétiser plus immédiatement ce vaste objectif, en instaurant un instrument de démocratisation relativement précis visant à relever les droits des travailleurs et ainsi garantir une plus grande inclusion sociale aux effets des processus de libéralisation économique, et leur meilleure participation à l'espace public. La consolidation démocratique est quant à elle beaucoup plus générale et plus facile à encourager qu'à concrétiser. Elle offre toutefois l'avantage de son imprécision et de son caractère non contraignant, probablement ce pourquoi il a été plus aisé de l'inscrire à l'agenda prioritaire des Amériques. La Déclaration de Principes du Sommet des Amériques de 1994 fait à plusieurs reprise référence à la complémentarité nécessaire que le projet veut établir entre prospérité, démocratie et plus grande justice sociale ${ }^{65}$. La Déclaration souligne à deux reprises l'importance d'encourager davantage la participation publique des individus et des organisations sur les plans politique, économique et social ${ }^{66}$, afin de mieux renforcer les processus démocratiques et aussi «garantir l'engagement et l'appui du public ${ }^{67} »$ au projet d'intégration hémisphérique. Le texte fait aussi mention deux fois des enjeux reliés à l'amélioration des normes de travail ${ }^{68}$, laquelle serait assurée par les moyens du libre-échange et de l'intégration économique accrue.

Le texte du Plan d'Action de Miami (décembre 1994) a été remanié à plusieurs reprises sur la question d'inclure ou non des dispositions claires relatives aux droits des travailleurs. Les États-Unis proposaient d'ajouter à l'Accord qui. régirait la ZLÉA des mesures précises couvrant le travail et l'environnement, s'inspirant du modèle des ententes parallèles établies dans le cadre de l'AlÉNA, en plus de l'ensemble des thèmes étudiés par les douze Groupes de travail de la ZLÉA qui ont enclenché leurs travaux à l'issue du

64. Larry DiAmond, "Consolidated Democracy in the Americas ", Annals of the American Academy of Political and Social Science, 550, 1997, pp. 12-41 ; Robert S. LeIkEN (dir.), A New Moment for the Americas, New Brunswick et Londres, Transaction Publishers, 1994; Howard J. WiARDA, "Consensus Found, Consensus Lost: Disjunctures in us Policy Toward Latin America at the Tum of the Century», Journal of Interamerican Studies and World Affairs, 39 (1), printemps 1997 , pp. 13-31. Voir aussi les propositions américaines présentées au Sommet de Miami reproduites dans «Excerpt of Draft us Cable on Miami Summit Initiative », Inside NAFTA, août 1994, p. 14: "Still, democratic institutions in many countries are weak and challenged to deliver the benefits of democracy. Progress in both political and economic reforms is in danger unless governments become more effective and responsive and thereby increase trust in public institutions. Government reform is at the top of the political agenda throughout the region, as leaders identify themselves with cleaner, more effective and efficient public administration. »

65. Sommet des Amériques (1994), Plan d'Action. Quatre objectifs généraux y sont identifiés à la première page: (i) préserver et renforcer la communauté de démocraties dans les Amériques; (ii) promouvoir la prospérité par le biais de l'intégration économique et du libre-échange; (iii) vaincre la pauvreté et la discrimination dans l'hémisphère; (iv) garantir le développement durable et protéger l'environnement.

66. Sommet des Amériques (1994), Déclaration de Principes, pp. 2 et 4.

67. Sommet des Amériques (1994), Déclaration de Principes, p. 4

68. Sommet des Amériques (1994), Déclaration de Principes, pp. 2 et 4. 
Sommet ${ }^{69}$. Le texte final du Plan d'Action n'a pas inclus la recommandation, mais indiquait toutefois, aux paragraphes portant sur le commerce ${ }^{70}$, que «le respect et la promotion des droits des travailleurs sera davantage renforcé au fur et à mesure de la progression de l'intégration économique ${ }^{71}$, ce qui laissait la voie à de multiples interprétations. Les négociateurs américains étaient néanmoins satisfaits de la formulation adoptée pour le Plan d'Action, laquelle établissait un seuil à partir duquel le sujet pouvait être développé davantage lors des négociations à venir ${ }^{72}$.

Depuis Miami, les ministres du Travail de l'hémisphère se sont réunis a dix reprises différentes pour étudier le dossier et acheminer leurs recommandations aux rencontres ministérielles annuelles sur la ZLÉA. Les rencontres des ministres du Travail ainsi que leurs travaux en cours, assistés par le Secrétariat de l'Organisation des États Américains (OÉA) en ce qui concerne le support logistique, se déroulent sur une base indépendante du processus de la ZLĖA. La rencontre des ministres est préparée par le Comité technique permanent pour les questions relatives au travail (COTPAL). Il n'y a aucun lien formel entre le travail du comité et les douze groupes de travail établis dans le cadre des négociations de la zlÉA. La dixième réunion du COTPAL, la dernière en liste date de mai 1997, a constitué l'occasion d'émettre un document recommandant essentiellement aux ministres du Commerce se réunissant à Belo Horizonte à la même date de considérer l'introduction d'une clause sociale aux négociations hémisphériques, tout en réaffirmant la complémentarité entre amélioration des normes de travail et libéralisations commerciales ${ }^{73}$. La Déclaration ministérielle de Belo Horizonte fait mention de la réception des recommandations émises par le corpal et renouvelle à cet égard l'engagement pris à Singapour dans le cadre de l'omc de respecter l'observation des normes fondamentales du travail identifiées à l'orr ${ }^{74}$.

69. La BID, la CÉPALC et l'ó́A sont chargées de fournir un soutien technique à l'élaboration des travaux des groupes de travail établis dans la foulée de la 2LEA. L'information, les statistiques commerciales et financières, ainsi que la publication des travaux des groupes de travail de la ZLEA est assurée par l'Unité au commerce de l'OÉA, qui soutient huit des douze groupes de travail de la ZLÉA, et la BID, qui en soutient quatre.

70. Sommet des Amériques (1994), Plan d'Action., «Libre-échange aux Amériques ", paragraphe Il (9) (2) du Plan d'Action, p. 7. Un aperçu des termes proposés par les États-Unis dans un document préparatoire est reproduit dans * Draft Leader's Declaration on Economic Issues at Miami Summit», Inside NAFTA, 10 novembre 1994 et « Nov. 29 Draft of Trade Language for Plan of Action », Inside NAFTA, 6 décembre 1994, p. 3.

71. Le texte exact est le suivant: «Au fur et à mesure de la progression de l'intégration économique de l'hémisphère, nous renforcerons davantage le respect et la promotion des droits des travailleurs, selon leur définition dans le cadre des conventions internationales appropriées. Nous nous abstiendrons d'adopter des restrictions commerciales déguisées, conformément aux obligations du GATT/OMC et aux autres obligations internationales. »

72. Selon les propos du Représentant au commerce Mickey KANTOR, reproduits dans « Officials Laud us Strategy for Achieving Trade Objectives at Summit», Inside NAFTA, 14 décembre 1994, pp. 14-15.

73. Declaration of the Tenth Interamerican Conference of Ministers of Labor, Dixième réunion du Comité technique permanent pour les questions relatives au travail, mai 1997, p. 6.

74. Sommet des Amériques, Déclaration ministérielle de Belo Horizonte, Troisième réunion des ministres du Commerce, 16 mai 1997, p. 4. 
Si, depuis Miami, les États-Unis ont proposé à plusieurs reprises d'inscrire une clause sociale dans l'accord-cadre qui devra régir la ZLEEA ${ }^{75}$, c'est toutefois à San José qu'ils ont recommandé pour la première fois par écrit l'inclusion d'une telle mesure, la rendant officielle. La quatrième réunion ministérielle à San José de mars 1998 a été l'occasion de débattre de nouveau du sujet et d'opposer un consensus négatif aux propositions américaines, appuyées par le Canada, d'établir un groupe d'étude sur les normes de travail. À l'issue de la réunion, il a été décidé de mettre sur pied un Comité des représentants gouvernementaux chargé de procéder à des consultations avec la «société civile», par l'intermédiaire d'organisations syndicales et sociales, d'institutions académiques et d'entreprises privées. Le statut et l'influence véritable d'un tel comité reste à clarifier, mais le seul fait de voir les pays en accepter la mise sur pied constitue déjà un progrès certain, ce qui prépare éventuellement la voie à des développements ultérieurs. Le paragraphe 17 de la Déclaration de San José portant sur la "participation de la société civile " comprend, en marge de l'établissement du Comité de représentants gouvernementaux, une brève annonce rappelant l'engagement pris par les ministres à Singapour. Cette annonce établissait leur volonté de respecter les normes fondamentales du travail établies dans le cadre de l'orr et de voir à ce que cette question soit traitée dans l'enceinte appropriée, c'est-à-dire en dehors des institutions économiques telle l'omc. Ce paragraphe a fait l'objet de remaniements majeurs, qui sont significatifs dans la mesure où la version finale qui a été signée à San José ne ressemble en rien aux propositions préliminaires qui avaient été soumises précédemment, notamment par le Costa Rica et surtout par les États-Unis. Le Costa Rica avait proposé lors de la réunion du Comité préparatoire de la zlÉA de la mi-février un paragraphe établissant clairement le fait que les questions de normes de travail n'allaient pas être traitées dans le cadre de la ZiÉA mais laissées aux travaúx de l'omc ${ }^{76}$. Le texte signé à San José ouvre aussi la voie à plus d'interprétations, en raison notamment du fait qu'il n'écarte pas la question des négociations futures en restant très général ${ }^{77}$. Les États-Unis proposaient de prendre officiellement acte de la Déclaration ministérielle à Singapour et des recommandations des ministres du Travail faites à Belo Horizonte afin d'établir un Groupe d'étude sur le travail chargé d'identifier les moyens de mieux garantir l'observance et la promotion des droits des travailleurs dans le processus d'intégration hémisphérique ${ }^{78}$. Le Groupe d'étu-

75. À Miami, la proposition avait été discutée sans faire l'objet d'une recommandation écrite, les chefs d'État et de gouvernement ayant manifesté leur désaccord à voir inclure des dispositions relatives au respect du droit de la main-d'œuvre dans les négociations sur la zLEA. Voir Inside NAFTA, 2 novembre 1994, p. 18.

76. Voir "Chair's Proposal of a Draft Ministerial Declaration of San Jose », Americas Trade, 5 février 1998, p. 20.

77. Sommet des Amériques (1998), Déclaration Ministérielle de San José, p. 5 : «We reaffirm our commitment to the principle of transparency of the negociation process, to facilitate the constructive participation of the different sectors of society. We also reaffirm our commitment to the Belo Horizonte Ministerial Declaration and to paragraph 4 of the Singapore Ministerial Declaration of the WTO. $\gg$

78. (Document préparatoire) Sommet des Amériques, «Draft of the Ministerial Declaration of San Jose», 16 mars 1998, p. 5. Voir aussi le Document préparatoire daté du 10 mars. 
des devait se réunir avant la fin du mois d'octobre 1998, être présidé par un des pays de l'hémisphère et fournir des recommandations avant la rencontre ministérielle prévue pour octobre 1999. D'autres pays ont également fait des propositions officielles sur la question. Le Mexique a recommandé de voir à développer un programme pour la migration des travailleurs au sein de l'hémisphère. La proposition n'a pas véritablement été discutée. Le Mercosur, présidé par l'Argentine pour la période qui couvre la rencontre ministérielle et le Sommet à Santiago, a recommandé l'établissement d'un Forum économique et social s'inspirant de son modèle. La proposition sera renouvelée à Santiago.

L'essentiel des discussions à San José a porté sur la proposition américaine plus que sur toute autre. Les représentants américains ont fait circuler plusieurs documents qui présentaient la position des États-Unis sur la question, dont un portait sur les « termes de référence » relatifs à la proposition. Ce document accordait cinq objectifs au Groupe d'étude: i) discuter des moyens les plus appropriés pour développer le dialogue entre les membres de la société civile et les instances politiques publiques sur les effets de l'intégration économique pour les travailleurs; ii) étudier davantage les propositions faites par les ministres du Travail afin d'y répondre officiellement; iii) compiler l'information sur les dispositions relatives à la main-d'œuvre déjà inscrites dans certains accords bilatéraux et intra-régionaux; iv) prendre note des activités menées à ce sujet au sein du cotpal et du prochain Sommet des Amériques afin de fournir des recommandations au Comité de négociations commerciales (TNC) de la ZLÉEA ${ }^{79}$.

Le document définitif adopté à San José au mois de févrirer 1998 répond fort modestement aux propositions américaines, qui sont une fois de plus reportées à des débats ultérieurs. On en reparlera lors du deuxième Sommet des Amériques à Santiago, en avril 1998, et plus significativement, lors de la cinquième rencontre ministérielle à Ottawa. Santiago n'a en fait rien résolu, la question n'ayant guère fait l'objet de discussions formelles. Même si les chefs d'État et de gouvernement y ont renouvelé leurs engagements respectifs en vue de renforcer les efforts communs au chapitre de la consolidation démocratique, l'essentiel des discussions au Sommet a plus directement porté sur les thèmes commerciaux qui ont maintenant éclipsé tous les autres sujets prévus à l'agenda de Miami. D'un autre côté, les organisations sociales et syndicales qui ont œuvré à développer des réseaux dans l'hémisphère, se sont rencontrées à Santiago peu avant le Sommet. Celles-ci ne semblent pas vouloir être laissées pour compte, pas plus maintenant qu'autrefois, ce qui rendra plus difficile d'ignorer l'opposition mieux organisée qui fragilise le support politique domestique en faveur d'une initiative de l'envergure du libre-échange des Amériques. Pour des questions de légitimité inteme donc, il y a peu de raisons de croire que le dossier social ait été clos à Santiago. En outre, la double présidence des États-Unis et du Brésil à la dernière ronde des négociations hémisphériques laisse à croire que les enjeux les plus controversés seront

79. (Document préparatoire), Réunion du Comité préparatoire de la zzėA, « us Proposal, Terms of Reference for Frad Study Group on Labor », San José, 10 février 1998. 
amenés à l'ordre du jour des discussions finales, avec l'effet qu'une clause sociale sera soit adoptée, l'administration américaine recourant à l'argument lourd de la contrainte interne imposée par le refus du Congrès de lui accorder l'autorité de contracter des accords commerciaux sans l'ajout de dispositions de réserves, soit remplacée par le forum économique et social à mandat consultatif que le Mercosur propose de son côté de mettre sur pied. Dans les deux cas, l'autonomie économique plus manifeste et l'importance politique des deux principaux acteurs de l'hémisphère ajoutera à leur capacité de négocier un accord qui puisse minimalement rencontrer leurs ambitions respectives, et qui détende également leurs propres pressions politiques internes. À ce chapitre, d'ailleurs, les États-Unis gagneraient à clarifier davantage la raison pour laquelle ils poussent autant à faire admettre une clause sociale aux négociations commerciales, en mettant l'accent sur le fait qu'une telle disposition permettrait, outre de confirmer leur rôle prédominant en matière de règles commerciales à respecter, surtout d'accroître le support politique interne en faveur du libre-échange pour asseoir sur des bases plus légitimes les décisions des chefs politiques de procéder à la libéralisation accrue des économies américaines, au sein des États-Unis comme ailleurs.

\section{Conclusion}

L'essentiel de la discussion qui précède réside dans l'idée que les ÉtatsUnis souhaitent, au moyen d'une clause sociale et de toute disposition qui pourrait plus ou moins y ressembler, mieux établir une relation de corrélation entre d'une part, libéralisme économique et d'autre part, démocratie ou, plus précisément, mécanismes de démocratisation. Pourquoi cela?

L'ambition de l'article était de répondre à cette question. Toutefois, notre projet a été partiellement réalisé. Nous avons surtout mieux mis en contexte la question des clauses sociales dans le cadre des stratégies américaines, selon nous complémentaires de l'unilatéralisme agressif et de la consolidation démocratique, posant l'interprétation principale voulant que, outre leur rôle central en matière de sanction commerciale et de formulation des règles relatives aux échanges, les États-Unis cherchent également à positionner le modèle démocratique comme prédominant. En somme, les explications hégémonistes cadrent avec l'approche que nous avons empruntée. Mais la question initiale n'a pas été épuisée pour autant et demanderait des développements supplémentaires que nous tenterons d'anticiper un peu.

Le projet américain d'adjoindre une clause sociale au commerce international a peu à voir avec le mythe de l'implication désintéressée des États-Unis dans le monde. Une clause sociale se révélerait par ailleurs fort certainement insuffisante à accélérer les mécanismes de démocratisation des systèmes politiques des pays en voie de développement. Par contre, la diffusion du fait que ce sont les États-Unis qui se proposent pour appuyer et mème se faire les premiers promoteurs du projet n'est pas sans intérêt. Toute campagne demande publicité, et l'on sait l'aspect exagéré qu'elle doit parfois prendre. En 
plus d'avoir pris le leadership économique au sortir de la guerre, les ÉtatsUnis ont depuis lors aussi prétendu verser dans la question du leadership politique, voire moral, du système politique international. Le fait de demander avec insistance qu'une disposition sociale soit inscrite à l'agenda multilatéral permet ainsi principalement aux États-Unis de se positionner comme les meneurs de file en termes de défense des droits sociaux internationaux, ce qui est entre parenthèses pour le moins étonnant, quand on connaît d'une part, l'hétérogénéité et la souplesse des législations sociales internes aux États-Unis et d'autre part, leur négligence notoire sur le plan de la ratification des traités internationaux portant sur ces questions. Mais cela l'est moins si l'on tient compte du fait que, principaux champions modernes du libre-échange, ils doivent également assurer la pérennité et donc la légitimation de ce qui avait été posé comme un ordre économique international il y a un peu plus de cinquante ans.

Ce processus de légitimation ne s'impose pourtant pas de soi. Il s'agit ni plus ni moins de continuer de rallier les membres de la société autour d'un consensus qui avait été établi sur la nécessité d'ouvrir les économies nationales et de mieux développer leur interdépendance pour éviter le recours au conflit. Il s'agit aussi dans une perspective plus domestique de convaincre tout un chacun des bienfaits de la «libéralisation », sur le plan de la croissance et de l'emploi, de façon à ce que les libéralisations puissent se poursuivre sans résistance sociale majeure. Mais pour ce faire, il convient qu'il y ait des canaux d'information, de consultation et de participation reliant la «société civile» aux différents appareils politiques, et que ces canaux soient dans un second temps bel et bien employés de part et d'autre. D'où la nécessité de démocratiser, à l'heure où la grande majorité des économies nationales participent à la grande kermesse commerciale mondiale et ce, quelles que soient leurs modalités d'organisation politique.

La démocratie, pour quoi faire? Des légions d'auteurs ont écrit la nécessité que le commerce, outre celui des armes bien sûr, puisse s'épanouir dans un climat de stabilité, sinon de paix. Les régimes démocratiques ont au moins depuis Kant été qualifiés par de nombreux auteurs, et malgré d'autres, comme plus pacifiques ${ }^{80}$. Mais plus fondamentalement encore: pour qu'il y ait commerce, il faut certes que des individus produisent et vendent, d'un côté, mais aussi et surtout que d'autres achètent. Et pour cela, il faut qu'ils en aient les moyens. Une clause sociale présenterait dans cette perspective simple l'intérêt de stimuler la création et la consolidation de classes moyennes capables de consommer et aussi d'assurer la plus grande stabilité des régimes politiques

80. Voir notamment Charles-Philippe DAvid et A. BeNESSAIEH, «La paix par l'intégration? Théories sur l'interdépendance et les nouveaux problèmes de sécurité», Études internationales, xxvill, n"2, juin 1997 
des pays en développements ${ }^{81}$, celles-ci participant à forger les consensus nécessaires à la cohésion nationale. Ces plus grandes stabilités domestiques respectives permettraient en dernière mesure de s'assurer du fait que le système économique international et le modèle économique libéral, voire aussi politique comme nous l'avons proposé dans notre lecture, soit là pour durer et ne saurait être profondément remis en question en raison de l'opposition diffuse ou organisée qu'il rencontre auprès des membres de la société qui s'en estiment exclus.

Il y a donc nécessairement une bonne distanciation entre le point de vue syndical, voulant qu'une clause sociale soit établie à des fins éthiques et dans certains cas, pour protéger davantage les travailleurs du Nord du marchandage de leurs avantages sociaux sous la pression de la concurrence venant des pays à bas régimes sociaux, et le point de vue des États-Unis, voulant plus pragmatiquement assurer au moyen de leur politique commerciale la continuité - et le leadership - de l'ordre économique posé aux lendemains de la guerre, sans pour autant permettre à tout un chacun de pratiquer une concurrence désordonnée réalisée avec les moyens du bord, dont ils sont touchés au premier rang compte tenu de l'ouverture de leur marché et aussi de l'ampleur de leurs activités exportatrices, qui doivent continuer de trouver preneur.

Cela dit, ce pragmatisme n'est pas clairement en opposition avec une autre perspective faisant que pour les États-Unis, la défense de la démocratie à travers le monde constitue un principe général, et selon certains de peu de foi, qui a souvent été invoqué pour justifier leurs interventions dans les affaires internes des nations. Toutefois nous croyons que ne peut être complètement évacuée l'idée que les États-Unis se sont depuis leur fondation attribué un certain rôle de missionnaire à travers le monde. Le projet d'inclure une clause sociale aux échanges internationaux a été par ailleurs fréquemment justifié de manière circulaire par les représentants et négociateurs commerciaux américains, faisant qu'il soit tout simplement «juste et bon » de le défendre ${ }^{82}$. Posant la liberté comme un idéal désirable et nécessaire, aux fondements de leur culture politique, les États-Unis se sont depuis l'après-guerre estimés les

81. Plusieurs auteurs ont en effet fait remarquer que l'absence ou la faible présence des classes moyennes était potentiellement source d'instabilité politique, en raison notamment des trop grands écarts entre la situation de la minorité, classe privilégiée économiquement et capable d'avoir accès aux sphères politiques, et celle de la majorité, dépourvue de véritable pouvoir économique comme d'accès directs à la machine gouvernementale.

82. Déclarations du président Clinton reprises aux pages 8 et 11 de l'introduction (Overview) du 1997 Trade Policy Agenda and 1996 Annual Report of the President of the United States on the Trade Agreements Program, Washington D. C., Government Printing Office, United States Trade Representative: * The Clinton Administration will continue its strong advocacy of the need to include worker rights (...). These issues are pressed because it is right to do so. 》 Voir aussi États-Unis, "Trade and Labor Standards ", dans Économic Report of the President, Transmitted to the Congress, Washington, février 1995, pp. 249-251. Les déclarations présidentielles accompagnant les publications annuelles de l'agenda commercial concernant la question des normes de la main-d'cuvre ont été répétées à chaque livraison de l'agenda depuis 1993. Voir pour chaque année, le texte reproduit dans le feuillet diplomatique Text, tel que Text (1993), «us Issues 1993 Trade Policy Agenda », 93-8, 3 septembre, p.1. 
principaux légataires de sa promotion à travers le monde, au niveau tant économique que politique ${ }^{83}$. Cette sorte de «bonté globale», voulant qu'ils se portent à la défense de la liberté là où elle est en danger, c'est-à-dire éventuellement partout, a aussi le délicat avantage de motiver par la vertu ce qui autrement apparaitrait parfois comme une autorité parfois injustifiée. Mais vertu ou idéaux et intérêts politico-économiques ne s'excluent pas nécessairement mutuellement, bien au contraire. Si le monde pouvait devenir cette civilisation unie par le credo libéral dont ils se veulent les porte-parole, établissant définitivement les principes de la liberté individuelle économique et politique au premier rang des valeurs universelles, les États-Unis n'en seraient pas plus mal et réaliseraient peut-être un vieux rêve absurde à la fois généreux et intéressé, la création d'une société mondiale libérale venant fixer l'espace marchand global sur des bases plus intégrées.

83. Jeffrey W. HAYES et Seymour Martin LIPSET, "The Social Roots of United States Protectionism ", dans BID/CÉPALC, Trade Liberalization in the Western Hemisphere, Washington, DC, 1995 , p. 499: «The ideals of freedom, liberty, and equality are seen as gospel, truths appropriate for all nations. Similarly, the American system of commerce must also be spread to societies seeking economic growth. Exporting the idea of market, of course, requires the idea that the us lead the way in unrestricted trade, a demand which was made of America only after World War li. " Voir aussi J. R. RugGie, "Third Try at World Order? America and Multilateralism After the Cold War », Political Science Quarterly, 109 (4), 1994, p. 555. 